\title{
Depletion of aneuploid cells in human embryos and gastruloids
}

\author{
Min Yang 1,2,5, Tiago Rito ${ }^{1,5}$, Jakob Metzger', Jeffrey Naftaly', Rohan Soman', Jianjun $\mathrm{Hu}^{2}$, \\ David F. Albertini ${ }^{(2)}{ }^{2}$, David H. Barad ${ }^{2,3}$, Ali H. Brivanlou ${ }^{1}{ }^{1 凶}$ and Norbert Gleicher $\left(\mathbb{1}^{1,2,3,4 凶}\right.$
}

\begin{abstract}
Chromosomal instability leading to aneuploidy is pervasive in early human embryos ${ }^{1-3}$ and is considered as a major cause of infertility and pregnancy wastage ${ }^{4,5}$. Here we provide several lines of evidence that blastocysts containing aneuploid cells are worthy of in vitro fertilization transfer. First, we show clinically that aneuploid embryos can lead to healthy births, suggesting the presence of an in vivo mechanism to eliminate aneuploidy. Second, early development and cell specification modelled in micropatterned human 'gastruloids' grown in confined geometry show that aneuploid cells are depleted from embryonic germ layers, but not from extraembryonic tissue, by apoptosis in a bone morphogenetic protein 4 (BMP4)-dependent manner. Third, a small percentage of euploid cells rescues embryonic tissue in mosaic gastruloids when mixed with aneuploid cells. Finally, single-cell RNA-sequencing analysis of early human embryos revealed a decline of aneuploidy beginning on day 3 . Our findings challenge two current dogmas: that a single trophectoderm biopsy at blastocyst stage to perform prenatal genetic testing can accurately determine the chromosomal make-up of a human embryo, and that aneuploid embryos should be withheld from embryo transfer in association with in vitro fertilization.
\end{abstract}

Although chromosomal mosaicism is common in human embryos derived from in vitro fertilization (IVF), most IVF centres consider the mosaic or aneuploid embryos to be abnormal, and thus withhold them from transfer into uteri ${ }^{6}$. Indeed, more than $80 \%$ of these embryos contain aneuploid blastomeres, while only $12.5 \%$ of fertilized oocytes are aneuploid; approximately $60 \%$ of embryos are euploid-aneuploid mosaic and only $22 \%$ are euploid ${ }^{8}$. The use of preimplantation genetic testing for aneuploidy (PGT-A) before embryo transfer to screen out aneuploidy has been proposed to improve delivery rates and reduce miscarriages in association with IVF $^{9}$ and has become an increasingly common clinical practice. Whether PGT-A improves IVF outcomes has, however, remained controversial $^{10-12}$. The high false-positive and false-negative rates in PGT-A means that a substantial number of diagnosed aneuploid or euploid embryos are actually mosaic ${ }^{13}$. As spontaneous and IVF pregnancies demonstrate similar levels of mosaicism (12\% versus $6.3 \%)^{14}$, excluding all embryos containing aneuploid cells from transfer would therefore deprive patients of options.

To test whether mosaic human embryos develop normally, we report on 32 women who underwent frozen-thawed embryo transfers with 77 blastocysts that were diagnosed as mosaic and aneuploid by PGT-A. Nine clinical pregnancies were established $(28.2 \%)$ from these transfers, 4 of which miscarried $(44.4 \%$ of pregnancies, $12.5 \%$ of cycles), and the remaining ones were delivered (15.6\%; Supplementary Table 1). Characteristics of participants and IVF cycles are presented in Supplementary Table 2. Median age was $40.6 \pm 4.1$ years. Our observed pregnancy and live birth rate matched or even exceed the national average of expected outcomes for age-matched individuals who did not use PGT-A diagnostics ( $18 \%$ pregnancy and $12 \%$ live birth rate at age 40$)^{15}$. Interestingly, while all transferred embryos were aneuploid, prenatal chromosomal analyses performed with chronic villus biopsies (CVS) or amniocenteses in delivered pregnancies uniformly revealed normal karyotypes. Comprehensive chromosomal analysis on the products of conception from three participants who miscarried in the first trimester showed that two demonstrated the same chromosomal abnormalities as previously detected in the original PGT-A analysis of the transferred embryos (Supplementary Table 1, patients 8 and 9), while the third (patient 7) had a normal karyotype, indicating the elimination of aneuploidy and suggesting a different cause for her miscarriage. Furthermore, successful cases of transferring diagnosed mosaic embryos have been reported ${ }^{16-18}$. Together, these clinical results unveil the developmental capacity of mosaic embryos, thereby supporting the possibility of a developmental rescue mechanism.

Indeed, an innate ability of the embryo to select against aneuploid cells has been demonstrated using a mouse model ${ }^{19}$. The mouse aneuploid cells of the inner cell mass (ICM) were eliminated by apoptosis, whereas trophectodermal aneuploid cells displayed proliferative defects but less apoptosis ${ }^{19}$. Even small percentages of euploid cells in the ICM resulted in normal euploid offspring ${ }^{19-21}$. However, in contrast to humans, spontaneous occurrence of aneuploidy is uncommon in mice, affecting less than $1 \%$ of embryos ${ }^{22}$. Therefore, a human model is needed to unravel the consequences of aneuploidy in early development and decipher the cellular and molecular mechanisms underlying the depletion or decrease of mosaicism during pregnancy.

In order to establish a development model for aneuploidy, we used RUES2 (NIHhESC-09-0013) human embryonic stem (ES) cells with reversine, a small molecule inhibitor of monopolar spindle 1 kinase that inactivates the spindle assembly checkpoint ${ }^{23}$. Reversine is known to cause severe chromosome segregation defects that efficiently generate aneuploidy in many cell types ${ }^{19,24,25}$. We confirmed the high efficiency of reversine treatment in generating aneuploid RUES2 cells using metaphase spreads; $94 \%$ of cells were aneuploid after $24 \mathrm{~h}$ of reversine treatment $(0.5 \mu \mathrm{M}$; Extended Data Fig. 1a-c). Reversine treatment yielded a karyotypically heterogeneous aneuploid cell population, with metaphase spreads exhibiting abnormal

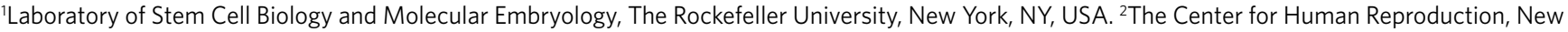
York, NY, USA. ${ }^{3}$ The Foundation for Reproductive Medicine, New York, NY, USA. ${ }^{4}$ Department of Obstetrics and Gynecology, Medical University of Vienna, Vienna, Austria. ${ }^{5}$ These authors contributed equally: Min Yang, Tiago Rito. e-mail: brvnlou@rockefeller.edu; ngleicher@thechr.com 
a

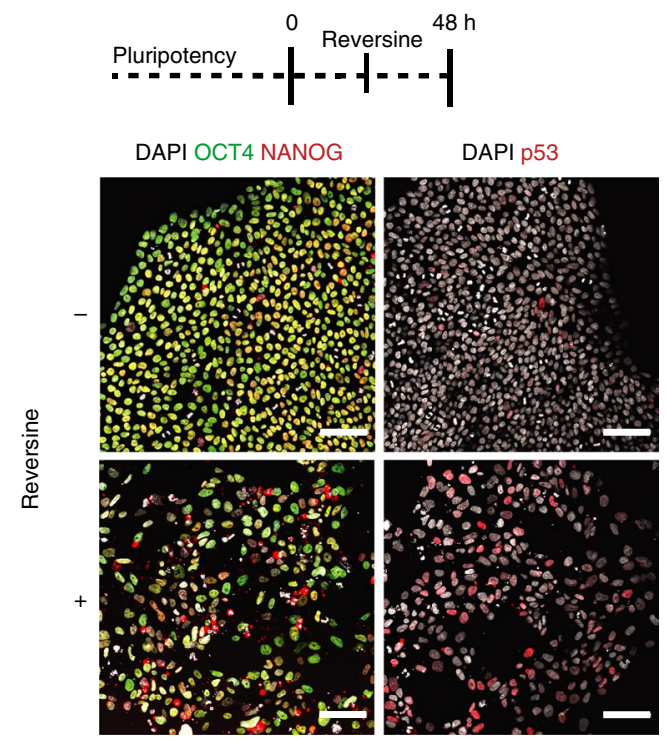

b
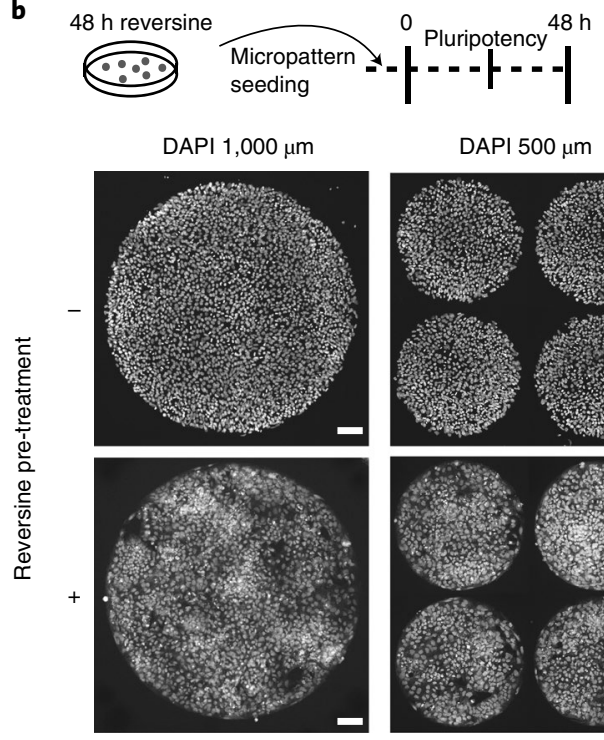

DAPI $500 \mu \mathrm{m}$
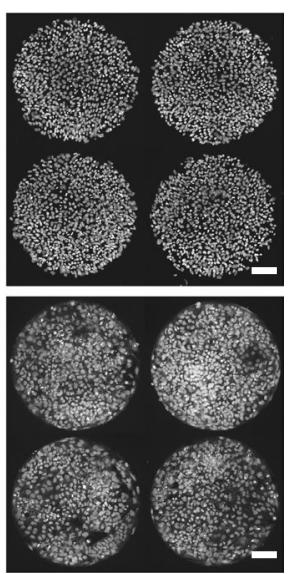

c

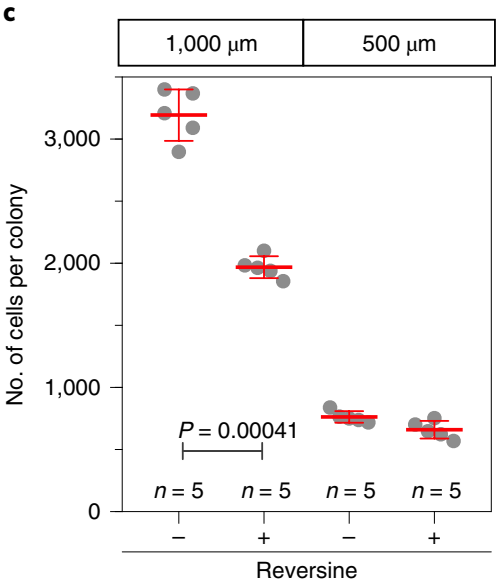

d

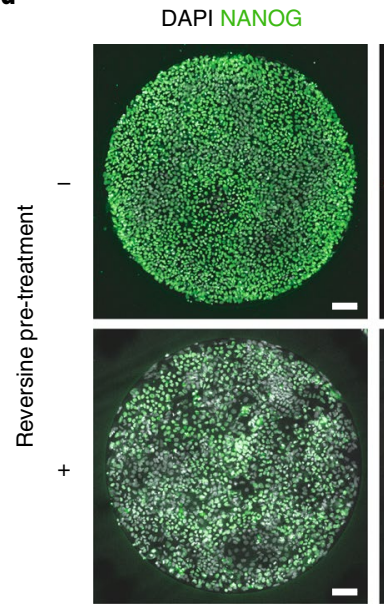

DAPI OCT4
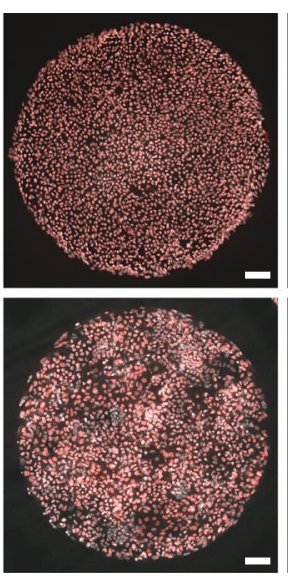

DAPI p53 CASP3
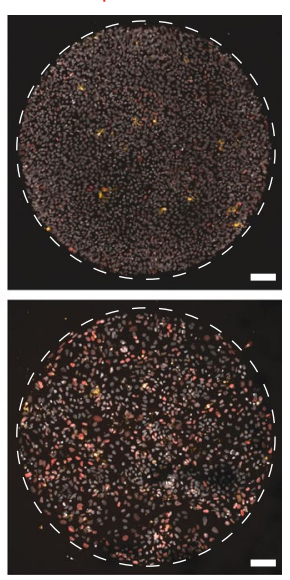

Fig. 1 | Induction of aneuploidy using reversine in RUES2 does not compromise the maintenance of pluripotency. $\mathbf{a}$, Treatment of RUES2 cells with $0.5 \mu M$ reversine for $48 \mathrm{~h}$ generates human ES cells with enlarged nuclei of irregular shape while maintaining pluripotency markers OCT4 and NANOG. Activation of p53 was observed. b, Colonies of reversine-treated and untreated RUES2 cells $48 \mathrm{~h}$ after seeding in pluripotency medium on $1,000 \mu \mathrm{m}$ and $500 \mu \mathrm{m}$ micropatterned coverslips. Compared with untreated RUES2 cells, reversine-treated RUES2 cells seeded on laminin-coated micropatterns give rise to more nonuniformly distributed cell colonies. c, Total cell number of each micropatterned colony of reversine-treated and untreated RUES2 cells after $48 \mathrm{~h}$ in the pluripotency medium. In micropatterned colonies $1,000 \mu \mathrm{M}$ in diameter, reversine-treated RUES2 cells are more numerous than untreated RUES2 cells. In micropatterned colonies of $500 \mu \mathrm{m}$ in diameter, there are no significant differences in cell number. Data are mean \pm s.d. $(n=5$ biologically independent cell colonies) and statistical significance was calculated using a two-tailed unpaired Student's $t$-test $\mathbf{d}$, Micropatterned colonies of reversine-treated and untreated RUES2 cells both expressed pluripotency markers OCT4 and NANOG after $48 \mathrm{~h}$ culture in pluripotency medium. Reversine-treated RUES2 showed increased expression of p53 (cell damage marker) but not CASP3 (cell death marker). a,b,d, Scale bars, $100 \mu \mathrm{m}$.

chromosome numbers with random nuclear fragmentation and abnormal chromosome crossovers. Karyotying showed that the parental RUES2 cell line exhibits a completely normal karyotype (Extended Data Fig. 1d), whereas the induced aneuploid cells comprise, but are not limited to, trisomies and monosomies (Extended Data Fig. 1e). Additionally, while remaining pluripotent, RUES2 cells treated with reversine for $48 \mathrm{~h}$ displayed an enlarged size and irregular nuclear shape, a morphology typically associated with aneuploidy ${ }^{26}$ (Fig. 1a and Extended Data Fig. 1f,g). We then cultured these reversine-treated cells in confined geometry on $500-\mu \mathrm{m}$ - and $1,000-\mu \mathrm{m}$-diameter circular micropatterned substrates for another $48 \mathrm{~h}$ under pluripotency conditions to further investigate their stability and physiology (Fig. 1b). The colonies of reversine-treated cells comprised fewer cells than the colonies of untreated cells after
$48 \mathrm{~h}$ of micropattern culture (Fig. 1c). Reversine-treated RUES2 cells exhibited increased expression of the DNA damage repair marker p53 but no difference in expression of the apoptosis marker CASP3 compared with untreated cells, and more importantly, the cells maintained the state of pluripotency, as indicated by expression of the pluripotency markers OCT4 (also known as POU5F1) and NANOG (Fig. 1d and Extended Data Fig. 1h).

We previously showed that gastruloids (models of human gastrulation derived from human ES cells) provide a robust and highly quantitative in vitro model to study early differentiation events with subcellular resolution and in real time ${ }^{27}$. When stimulated with BMP4, RUES2 cells self-organize and differentiate to generate embryonic and extraembryonic fates that are patterned in radially symmetrical domains with ectoderm at the centre, extraembryonic 
a
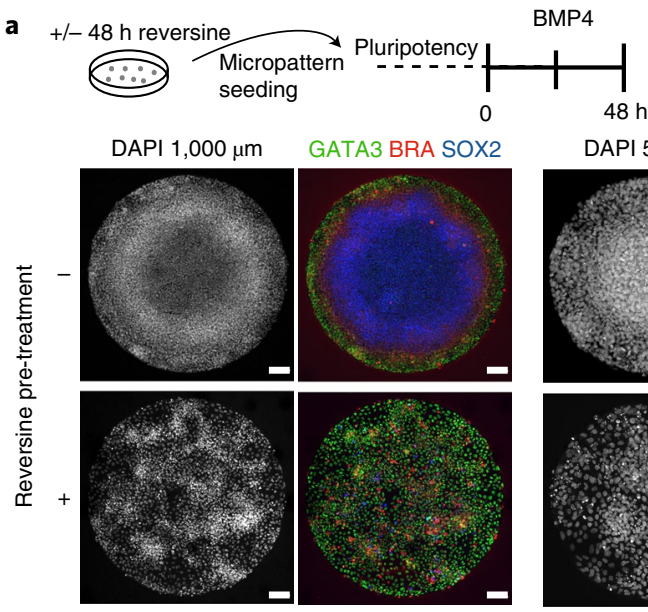

$48 \mathrm{~h}$

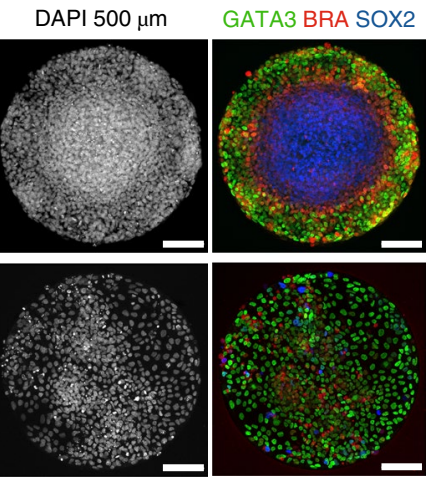

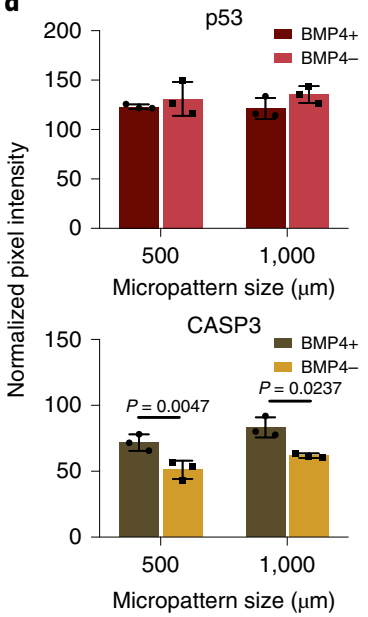

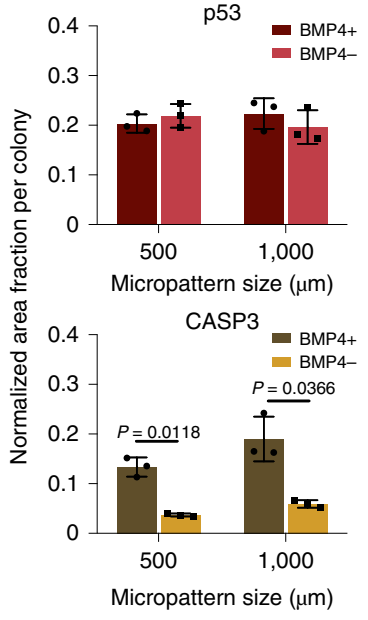

BMP4+
BMP4- b

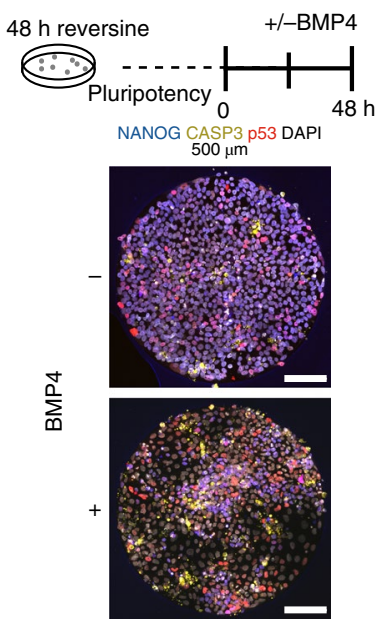

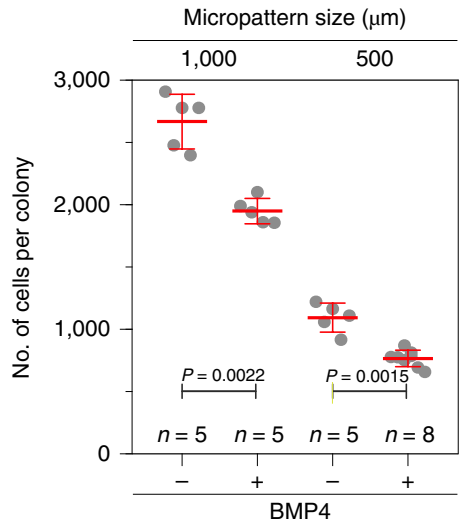
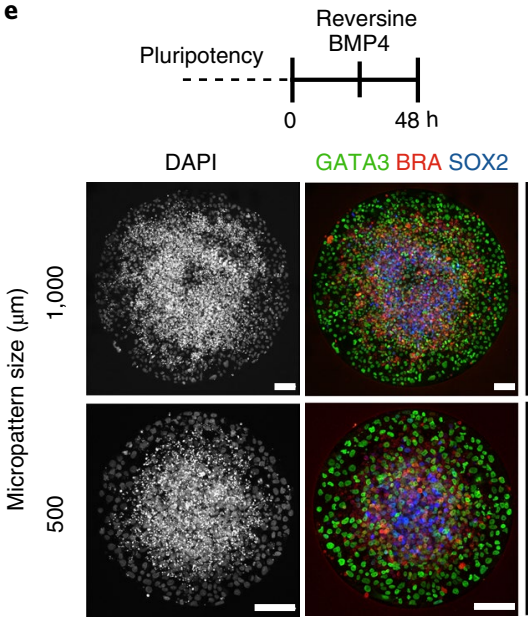

CASP3 p53 DAPI

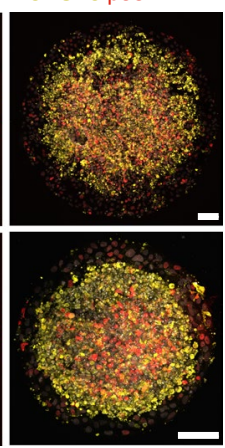

Fig. 2 | Gastruloids self-organization reveals lineage-specific behaviours and BMP4-dependent apoptosis of aneuploidy. a, RUES2 cells were seeded on laminin-coated micropatterns and cultured with $50 \mathrm{ng} \mathrm{ml} \mathrm{m}^{-1}$ BMP4 for $48 \mathrm{~h}$ to create radially organized domains of the germ layers (SOX2+ for ectoderm, $\mathrm{BRA}+$ for mesoderm and GATA3+ for trophectoderm) mimicking embryonic gastrulation (gastruloids). The gastruloids derived from reversine-treated aneuploid RUES2 were depleted of the ectoderm centre and mesoderm ring, and extraembryonic cell fate was not affected in either $1,000 \mu \mathrm{m}$ or $500 \mu \mathrm{m}$ micropatterned substrates. b, Micropatterned colonies were derived from reversine-treated aneuploid RUES2 cells with or without $48 \mathrm{~h}$ BMP4 induction. BMP4 induction decreases expression of the pluripotency marker NANOG and increases the activation of CASP3. c, Quantification of total cell numbers of each reversine-treated aneuploid micropatterned colony treated with or without BMP4. In both 1,000 $\mu \mathrm{m}$ and $500 \mu \mathrm{m}$ micropatterned substrates, a significant difference in cell number was observed following BMP4 treatment. $n=5$ biologically independent cell colonies. d, Normalized fluorescent pixel intensity and area fraction were quantified for p53 and CASP3 on micropatterned colonies. BMP4 induction increased the fluorescence intensity and area fraction of CASP3 staining in colonies derived from reversine-treated RUES2 cells. p53 levels displayed no notable difference between colonies with and without BMP4 induction. $n=3$ biologically independent cell colonies. e, RUES2 cells were seeded on the micropatterns and treated with $0.5 \mu M$ reversine during the 48h BMP4 induction. Embryonic germ layers (SOX2+ and BRA+) were induced together with the extraembryonic layer (GATA3+) but were less compact in the gastruloid. Activation of the cell death marker CASP3 and the cell damage marker ( $p 53$ ) concentrated at the SOX2-BRA territory, where DAPI staining shows a dense population of nuclei fragments. a,b,e, Scale bars, $100 \mu \mathrm{m}$. c,d, Data are mean \pm s.d.; two-tailed Student's $t$-test.

and endoderm at the edge and mesoderm in between. In the extraembryonic territory, cells express markers of early trophectoderm that are expressed in day 5 embryos (for example, CLDN4, SLC7A2 and TACSTD2), thus modelling aspects of preimplantation development, including that of trophectodermal lineages ${ }^{28}$. To probe the effects of aneuploidy at these critical stages of development, aneuploid RUES2 gastrulpoids generated with reversine treatment were cultured in $1,000 \mu \mathrm{m}$ and $500 \mu \mathrm{m}$ micropatterned colonies and then stimulated with BMP4. In response to BMP4, almost all cells in the aneuploid gastruloid colonies were positive for GATA3, suggesting global conversion or differential survival of extraembryonic tissue (Fig. 2a). SOX2+ and BRA+ domains were largely absent. We further verified the extraembryonic identity of the remaining GATA3+ cells in the aneuploid gastruloids, which showed expression of CDX2 and TFAP2A at the protein level as well as nuclear YAP staining, a characteristic of trophectoderm ${ }^{29,30}$ (Extended Data Fig. 2a). We demonstrated previously that cell density has a strong influence on the self-organization of gastruloids ${ }^{31}$. To investigate whether the loss of patterning in reversine-treated aneuploid gastruloids was caused by low cell density, we generated compact aneuploid micropatterned colonies by either directly seeding twice as many cells or allowing cells to divide further to reach a density comparable to or higher than that of control untreated colonies before stimulation. Increasing density had no effect on the observed patterning phenotype, thus eliminating the possibility of density-related effects (Extended Data Fig. 2b,c). 
a
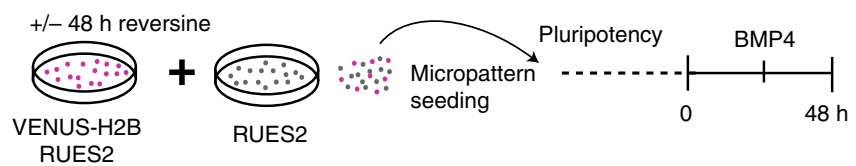

RUES2

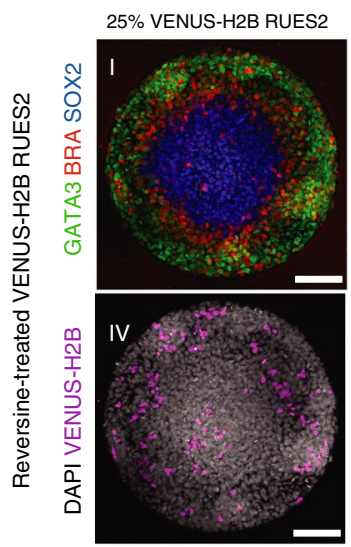

$50 \%$ VENUS-H2B RUES2

75\% VENUS-H2B RUES2
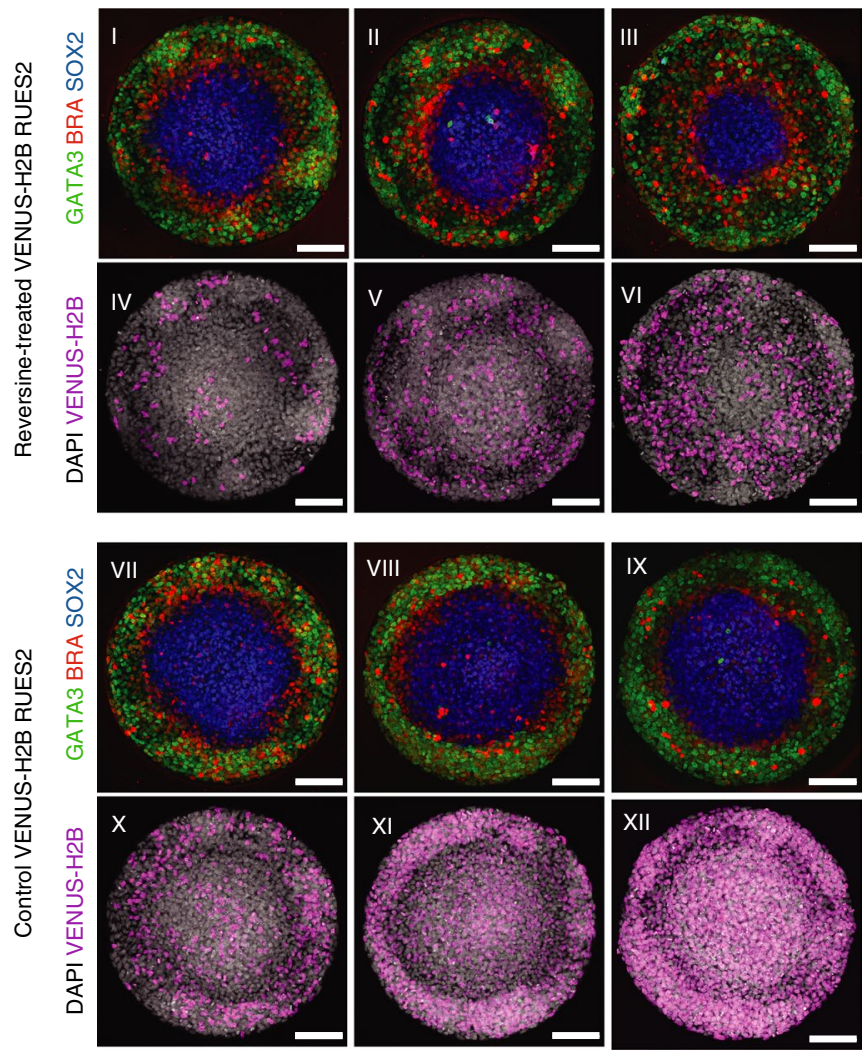

b
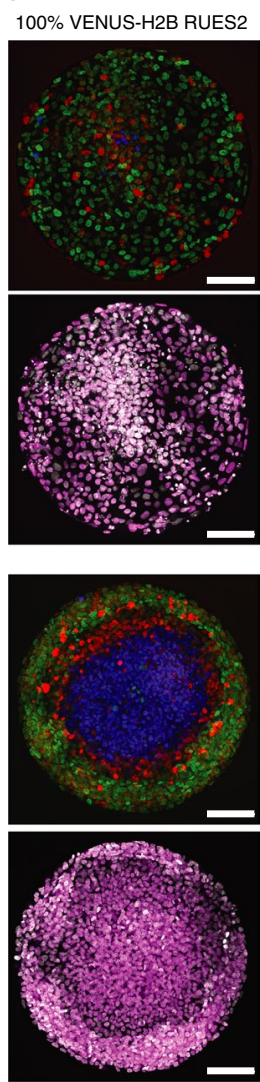

c

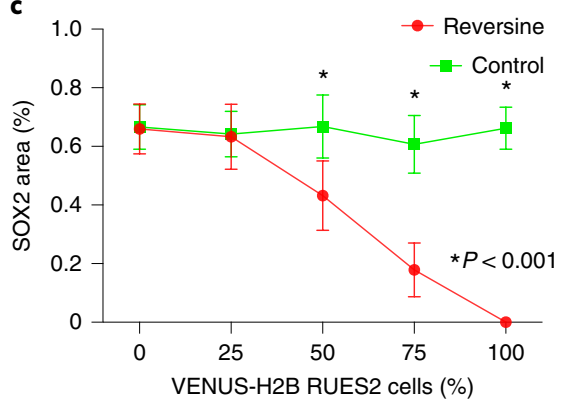

d
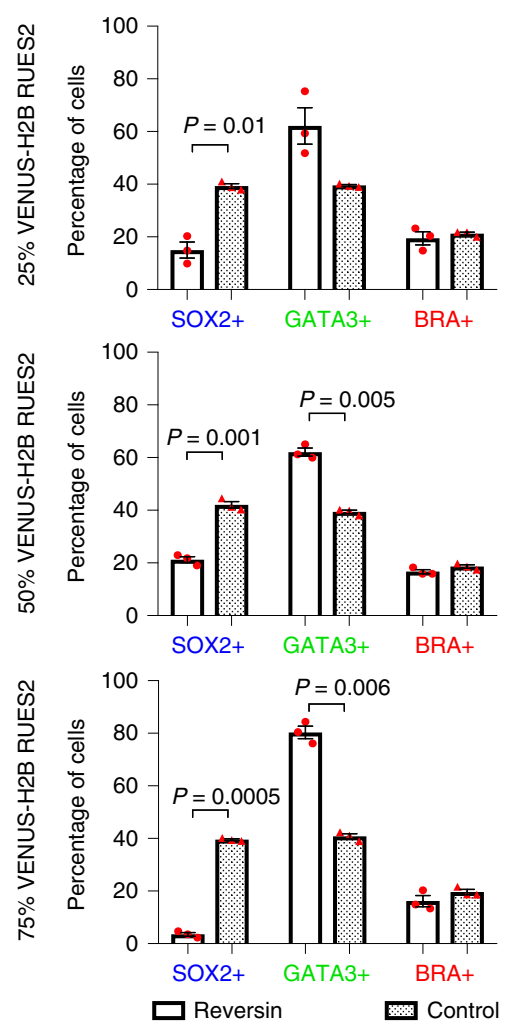

Fig. 3 | Complete cell lineages were successfully developed in mosaic gastruloids, and aneuploid cells were preferentially allocated to the trophectoderm. a, Reversine-treated cell-tracking line VENUS-H2B-RUES2 was mixed with untreated non-tagged RUES2 cells with a ratio of 1:3, 1:1 and 3:1 (25\%,50\% and 75\% VENUS-H2B-RUES2 cells, respectively) and seeded on micropatterns for BMP4 induction (I-VI). In control micropatterned colonies, VENUS-H2B-RUES2 were not treated with reversine, and radially organized domains of germ layers (SOX2+ ectoderm, BRA+ mesoderm and GATA3+ trophectoderm) were formed, and VENUS-H2B-RUES2 cells were evenly disturbed in the gastruloids (VII-XII). The 25\%, 50\% and 75\% mosaic gastruloids were able to generate embryonic layers (SOX2+ and BRA+) and the extraembryonic layer (GATA3+) after BMP4 induction. However, the size of the centre embryonic domain shrunk with the increase in the ratio of aneuploid cells, indicating fewer cells differentiated into ectoderm fate. $\mathbf{b}$, Gastruloids derived from 100\% VENUS-H2B-RUES2. c, The size of the centre ectoderm (SOX2+) for gastruloids with different percentages of VENUS-H2B-RUES2, obtained from immunofluorescence data and plotted as a percentage of total gastruloid size. $n=15$ biologically independent colonies for each control group, $n=16$ (0\% VENUS-H2B-RUES2 cells), 23 (25\%), 28 (50\%), 23 (75\%) and 20 (100\%) for reversine-treated groups. d, VENUS-H2B-RUES2 cells were quantified for different lineages using immunofluorescence data. Compared with the control gastruloids, the reversine-treated $V E N U S-H 2 B-R U E S 2$ cells in the mosaic gastruloids accounted for a significantly lower percentage of ectoderm cells (SOX2+) and a higher percentage of trophectoderm cells (GATA3+), indicating that aneuploid cells preferentially differentiated into trophectoderm cell fate. $n=3$ biologically independent colonies. This difference is more pronounced in the gastruloid with a higher percentage of aneuploid RUES 2 cells. a,b, Scale bars, $100 \mu M$. c,d, Data are mean \pm s.d.; two-tailed Student's t-test.

To evaluate the extent to which differentiation plays a role in the survival from aneuploidy, we compared micropatterned colonies derived from reversine-treated aneuploid RUES2 cells with and without $48 \mathrm{~h} \mathrm{BMP} 4$ stimulation. In the absence of BMP, the colonies remained pluripotent, exhibiting high density and without an obvious pattern of widespread cell death (Fig. 2b). Despite seeding the same quantity of cells initially, we detected significantly lower cell numbers after $48 \mathrm{~h}$ of BMP4 treatment in the colonies derived from reversine-treated RUES2 (Fig. 2b,c), indicating that cell death during gastruloid formation is not a consequence of reversine but of induced BMP-stimulated differentiation. In response to BMP4, aneuploid RUES2 cells differentiated with prompt downregulation of pluripotent marker NANOG in the majority of cells and an increase in cell death marker CASP3 in gastruloids (Fig. 2b,d). 
To further evaluate how aneuploid cells perform compared with euploid cells following treatment with BMP4, fluorescently tagged reversine-treated aneuploid (RFP-H2B-RUES2, pink) and untreated control euploid (VENUS-H2B-RUES2, green) cells were mixed in a 1:1 ratio in the same micropatterned colonies and cultured for $48 \mathrm{~h}$ either with or without BMP4. Live-cell imaging showed that, while the euploid control RUES2 predominate in both conditions, reversine-treated cells were more severely depleted in the colony that was induced by BMP4 (Supplementary Video 1, right) compared with non-induced colonies in pluripotency medium (Supplementary Video 1, left). Therefore, differentiation (that is, escape from pluripotency) reduces cell tolerance towards aneuploidy. To address cell-type specificity in the BMP4-dependant apoptosis, we performed an experiment addressing the consequence of inducing aneuploidy after allowing BMP4 to begin differentiation. We reasoned that since reversine takes at least one cell cycle to generate aneuploidy, and BMP4 transduces signals within less than $1 \mathrm{~h}$, differentiation will be induced before aneuploidy exert its effects. Consistent with specification occurring before induction of aneuploidy, the cells of embryonic germ layers (SOX2+ and BRA+) were induced together with GATA3+ layer, in concentric rings. However, examination of p53 and CASP3 revealed a significant enhancement in the SOX2+BRA+ territory (Fig. 2e). This result demonstrates that aneuploidy-induced cell death exhibits lineage preference during early specification, and that the GATA3+ extraembryonic-like cell population is particularly resilient to aneuploidy.

These lineage-specific findings parallel studies in mouse embryos that detected a higher frequency of apoptosis in aneuploid cells in ICM than in trophectoderm, where reversine was also used to induce aneuploidy ${ }^{19,32}$. As aneuploidy in mouse embryos is rare compared with human embryos, the fact that aneuploidy can be depleted in vivo suggests evolutionary conservation of this embryonic activity. Notably, both pluripotent epiblast and differentiated trophectoderm cells tolerated aneuploidy and displayed significantly lower levels of apoptosis, suggesting the presence of a similar underlying mechanism. As pluripotent cells have been shown to lack key regulators of the cell cycle, which allow the bypassing of checkpoints, the tolerance of trophectoderm may also be due to cell cycle checkpoint regulation similar to the one in pluripotency ${ }^{33}$. Further studies of trophectodermal cell cycle checkpoints will clarify this possibility.

To probe the putative rescue mechanism during mosaic embryo development, we constructed a mosaic gastruloid model and investigated whether the presence of euploid cells can rescue the lineage formation and the spatial patterning of aneuploid gastruloids. To this end, we treated VENUS-H2B-RUES2 cells with reversine and mixed the treated cells at different ratios with non-tagged RUES2 cells to generate mosaic gastruloids (Fig. 3a (I-VI)). We used three ratios of reversine-treated VENUS-H2B RUES2:non-tagged RUES2 cells: 1:3 (25\% aneuploid cells; Fig. 3a (I and IV)), 1:1 (50\% aneuploid cells; Fig. 3a (II and V)) and 3:1 (75\% aneuploid cells; Fig. 3a (III and VI)). As the control, we used the same mixing ratio to combine untreated
VENUS-H2B-RUES2 with non-tagged RUES2 (Fig. Fig. 3 (VIIXII)). When aneuploid cells comprised $25 \%$ of the cell population, the gastruloids exhibited self-organization of normal spatial patterning and complete cell lineages compared with controls (Fig. 3a (I and IV) versus Fig. 3a (VII and X)). Gastruloids with 100\% reversine-treated or untreated VENUS-H2B RUES had the same phenotypes as those with the parental RUES2 cell line (Fig. 3b). Surprisingly, the mosaic micropatterned colonies containing 50\% or $75 \%$ of aneuploid cells were also able to form gastruloids with normal self-organization of embryonic and extraembryonic germ layers (Fig. 3a (II, III, V and VI)). However, we note that the SOX2+ territory decreases in size with increasing aneuploidy ratio (Fig. $3 \mathrm{c}$ ). Characterization and quantification of the cells in mosaic gastruloids revealed a significantly lower percentage of aneuploid SOX2+ cells $(18.32 \%, 15.79 \%$ and $27.16 \%$ lower for $1: 3,1: 1$ and $3: 1$ ratios, respectively). Of note, a significantly higher percentage of aneuploid cells in the mosaic gastruloid contributed to GATA3+ extraembryonic cells $(16.90 \%, 17.22 \%$ and $30.02 \%$ higher for $1: 3,1: 1$ and $3: 1$ ratios, respectively) (Fig. $3 \mathrm{~d}$ ). The tendency of aneuploid cells to acquire extraembryonic fate seems more pronounced if the mosaic gastruloids contain fewer euploid cells, suggesting the existence of a threshold of depletion. Together, our results demonstrate that even a small fraction of euploid cells in the mosaic gastruloid is sufficient to generate normal self-organizing radial patterns, with aneuploid cells being preferentially allocated to the trophectodermal layer.

Our clinical and gastruloid data suggest the existence of an apoptotic depletion mechanism for aneuploid cells in embryos that depends on their differentiation trajectory and timing. We sought to probe the timing of the depletion of aneuploid cells by investigating its prevalence in early human embryos. We analysed two existing single-cell RNA-sequencing (scRNA-seq) datasets from a total of 101 embryos $^{34,35}$. The chromosome-wide analysis was used to identify gross abnormalities in gene expression and infer aneuploidy from scRNA-seq data using a strategy similar to the one described by Griffiths et al. ${ }^{36}$ (Fig. 4a). The data from Petropolous et al. analyse 1,529 cells from 88 preimplantation embryos ${ }^{34}$ collected from embryonic day 3 (E3) to E7, while the data from Zhou et al. analyse of 5,911 cells from 21 embryos from E6 to E14, where cells from day 8 and beyond were collected from in vitro attached embryos ${ }^{35}$. For each chromosome in a cell, we calculated a normalized sum of expression levels across all genes and converted it to a $z$-score. When $z$-score values for the X chromosome between E3 and E7 were calculated ${ }^{34}$, two distinct cell populations were observed at E3-E5, reflecting the existence of both male and female cells. These populations subsequently merged into a single cluster by E6-E7 (Fig. 4b), indicating the inactivation of one $\mathrm{X}$ chromosome in female cells during preimplantation stages ${ }^{34}$. Since even in this extreme case of variation in sex chromosome gene expression, the $z$-score values were confined between \pm 3 , values outside this range are probably due to aneuploidy. After identifying aneuploidy in single cells, we calculated the percentage of aneuploidy both for each day and, independently, for the two datasets to avoid any bias introduced by

Fig. 4 | Detection of aneuploidy in human embryos using scRNA-seq. a, Schematic showing the strategy of using existing human embryo scRNA-seq data to estimate prevalence of aneuploidy. $\mathbf{b}$, Threshold values of $z$-score used to call aneuploidy in the context of $X$ chromosome inactivation. Dots represent individual cells. Two distinct cell populations are visible on E3-E5, which subsequently merged into one cluster by E6 as the inactivation of one $X$ chromosome in the female cells reduces the total chromosome expression to values close to those of male cells during the preimplantation stage. As even in this extreme case of chromosome gene expression variation, the $z$-score values are confined within $|3|$, we reasoned that values above this threshold are the result of aneuploidy. $\mathbf{c}$, Distribution of $z$-score values of each chromosome per cell for the two published scRNA-seq datasets: from Petropoulos et al. ${ }^{34}$ (top), containing 1,529 cells from 88 E3-E7 embryos, and from Zhou et al. ${ }^{35}$ (bottom), with 5,911 cells from 21 E6-E14 embryos. Red dots highlight putative aneuploid chromosomes with z-score $>|3|$. d, For all analysed cells, high aneuploidy rates were observed at the earlier preimplantation stages, with a sharp decline at E4-E7. The aneuploidy rate was persistently low up to E14. e, Aneuploidy ratio per embryo was highly variable and high in the early stages, with rapid decline at E6. Fully aneuploid embryos (100\% cells probed) ceased to be detected from E6 onwards. $n=13$ (E3), 16 (E4), 24 (E5), 18 (E6) and 17 (E7) for Petropoulos et al. ${ }^{34}$ data, and $n=4$ (E6), 5 (E8), 10 (E10), 4 (E12) and 1 (E14) for Zhou et al. ${ }^{35}$ data. In box plots, the centre line represents the median, + indicates mean, boxes extend from the 25th to 75th percentiles and whiskers show the range of values. 
NATURE CELL BIOLOGY

LETTERS

technical variance (Fig. Ac). A high aneuploidy rate $(81.82 \%)$ was observed in preimplantation embryos at E3, with a sharp decline starting from E4-E5. The aneuploidy rate decreased to $5.36 \%$ by E7. Our analysis showed that data from both datasets demonstrate consistent aneuploidy rates from E6 to E8, while the data from Chou et al. ${ }^{35}$ additionally displayed a persistently low aneuploidy rate up to E14 (Fig. Ad). Consistent with the cell aneuploidy rates, the anewploidy ratio per embryo was highly variable, being more abundant in the early stages, and showed a decline over time. On day 3 , every embryo in the dataset was more than $50 \%$ aneuploid, whereas the aneuploidy rate in mosaic embryos decreased from E3 to E6, with the median aneuploidy rate decreasing from $83.33 \%$ to $11.1 \%$ from E3 to E4. Embryos with $100 \%$ aneuploid cells were not present after E6, suggesting their lack of potential for survival (Fig. He). Overall, our analysis shows that aneuploidy is pervasive in human embryos, but the prevalence of aneuploid cells in mosaic embryos a

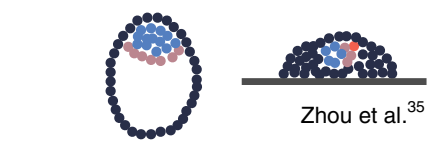

Petropoulos et al. ${ }^{34}$

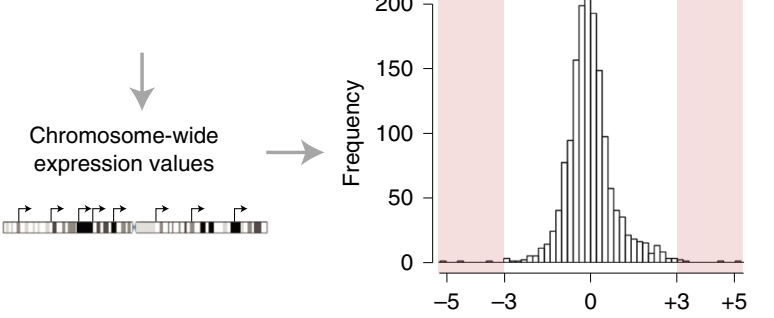

Chromosome $z$-score per cell b

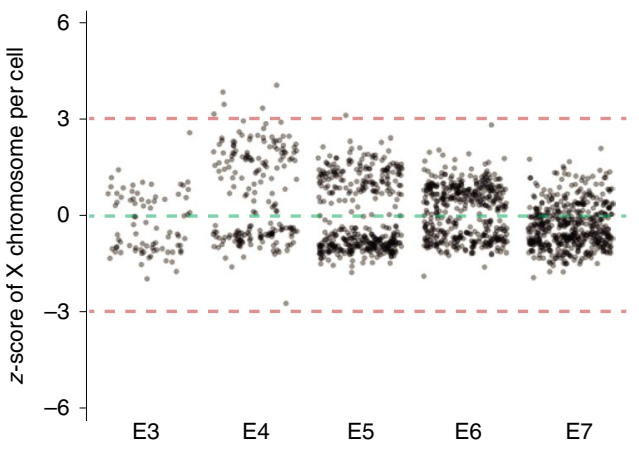

c
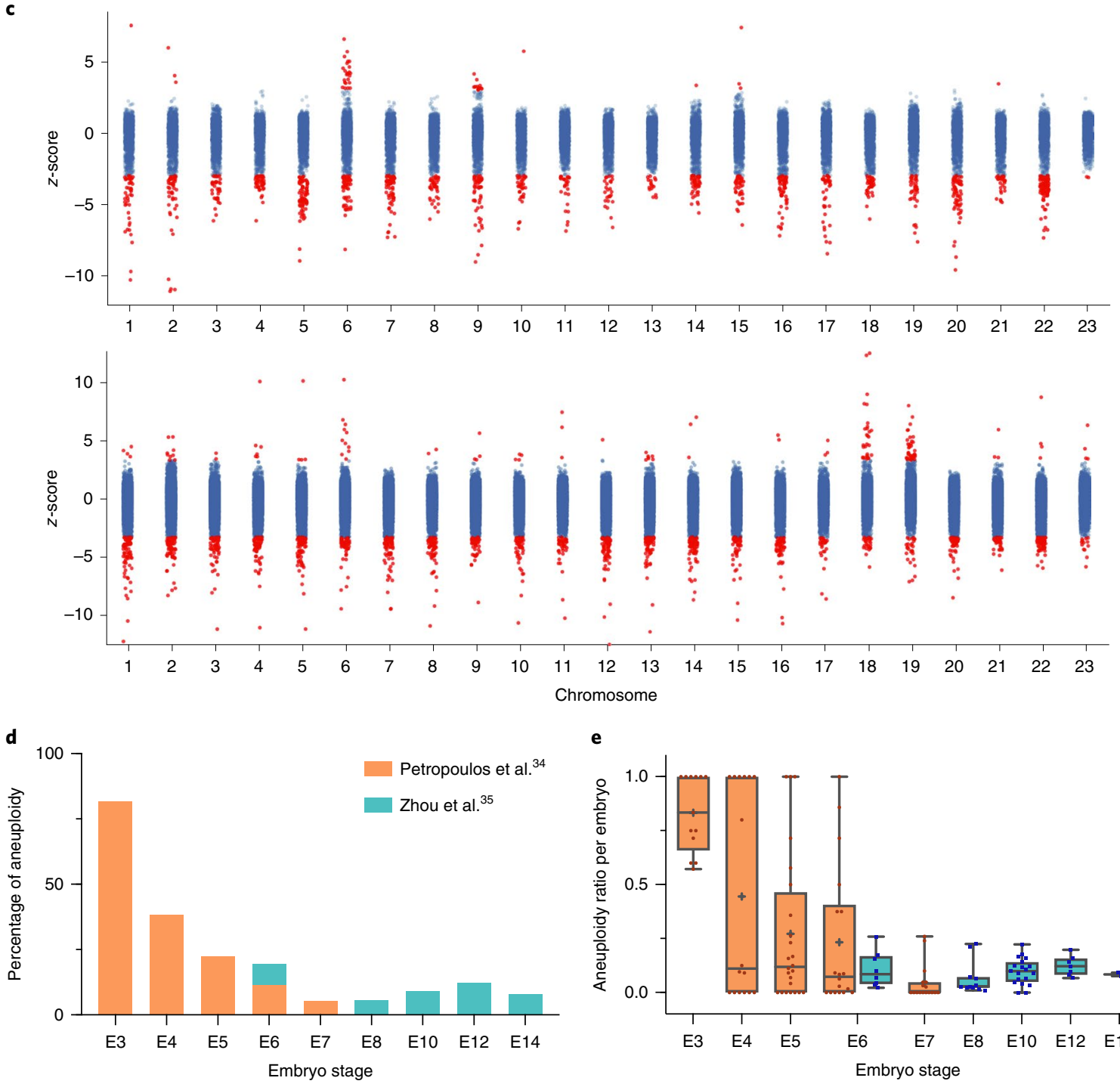

e

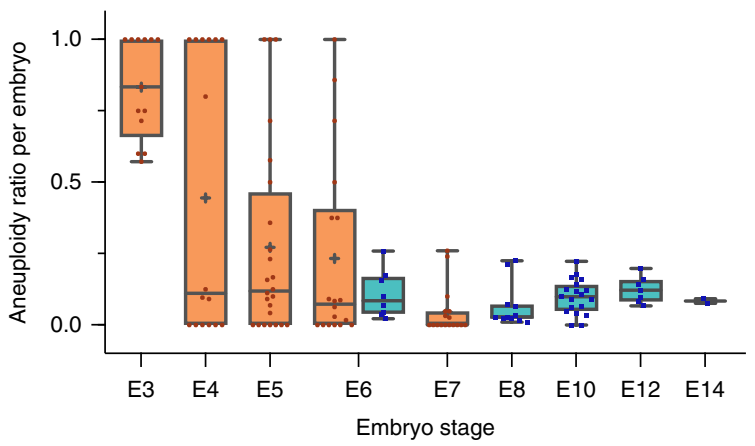

NATURE CELL BIOLOGY | VOL 23 | APRIL 2021 | 314-321 | www.nature.com/naturecellbiology

319 
progressively declines, suggesting a mechanism that eliminates aneuploidy similar to the one that we have described in gastruloids.

Recent bioinformatic analysis of scRNA-seq data from in vitro cultured human post-implantation embryo further revealed a higher tolerance to aneuploidy in the trophectoderm lineage than in primitive endoderm and epiblast lineages derived from $\mathrm{ICM}^{37}$. The enrichment of aneuploidy tolerance in trophectoderm compared with other lineages becomes more extreme as development proceeds. Based on our gastruloid data, which recapitulated this lineage-specific observation, we propose that aneuploid cells in mosaic embryos tend to survive in the trophectoderm, with little influence on ICM development. This could explain why confined placental mosaicism (CPM) is common, occurring in at least $2 \%$ of ongoing natural pregnancies ${ }^{38}$, and why the prevalence of genetic mosaicism is low in postnatal DNA samples ${ }^{39}$. A recent study showed that chromosome instability frequently occurs in both naturally conceived and IVF pregnancies and that the prevalence decreases at later stages of prenatal development ${ }^{14}$. Some aneuploid cells in early-cleavage-state embryos were segregated into placental lineage only ${ }^{14}$. This in vivo finding offers strong evidence in support of our proposal. Given that studies have demonstrated that $\mathrm{CPM}^{40-43}$ does not appear to be associated with adverse pregnancy outcomes, and that an extensive load of copy number variations was detected in human placenta ${ }^{44}$, aneuploidy in the extraembryonic lineage should not necessarily be viewed as abnormal. The tolerance towards chromosome instability in extraembryonic tissue, like that seen in cancer, might be associated with rapid proliferation, migration and invasiveness. The presence of aneuploidy in trophectoderm of blastocyst-stage embryos may, therefore, have physiological functions.

In sum, this study presents a comprehensive picture of aneuploid cell fate in early human development. Our results directly demonstrate the developmental potential and robust aneuploidy depletion ability of mosaic embryos, thus challenging the clinical relevance of ploidy analysis in trophectoderm biopsies as part of routine IVF practice. Gastruloids that recapitulate early embryonic development serve as a competent in vitro model for probing the underlying mechanism of the observed aneuploidy elimination in human embryos. To our knowledge, lineage-specific behaviours of aneuploid cells in humans have not previously been examined with quantification at the cellular level. Analysis of the available embryo single-cell data also suggests an aneuploidy elimination mechanism similar to the one described in gastruloids. Furthermore, the effects of BMP4-induced differentiation in aneuploid human ES cells presented in this study pave the way to future studies on the role of BMP signalling in aneuploidy depletion, and on the precise order and timing of events, particularly how the gastruloid results relate to the earlier aneuploidy depletion inferred from currently available single-cell transcriptomics. Additional studies using more targeted strategies that generate aneuploidy with defined karyotypes will be required to further address the molecular basis of aneuploidy depletion. Newly developed 3D models of a human epiblast will also provide methods to investigate embryonic aneuploid cell fate acquisition in a spontaneously symmetry-breaking model of human embryogenesis ${ }^{45}$.

\section{Online content}

Any methods, additional references, Nature Research reporting summaries, source data, extended data, supplementary information, acknowledgements, peer review information; details of author contributions and competing interests; and statements of data and code availability are available at https://doi.org/10.1038/ s41556-021-00660-7.

Received: 21 May 2020; Accepted: 4 March 2021; Published online: 9 April 2021

\section{References}

1. Delhanty, J. D. et al. Detection of aneuploidy and chromosomal mosaicism in human embryos during preimplantation sex determination by fluorescent in situ hybridisation, (FISH). Hum. Mol. Genet. 2, 1183-1185 (1993).

2. Angell, R. R., Aitken, R. J., van Look, P. F. A., Lumsden, M. A. \& Templeton, A. A. Chromosome abnormalities in human embryos after in vitro fertilization. Nature 303, 336-338 (1983).

3. McCoy, R. C. Mosaicism in preimplantation human embryos: When chromosomal abnormalities are the norm. Trends Genet. 33, 448-463 (2017).

4. Macklon, N. S., Geraedts, J. P. M. \& Fauser, B. C. J. M. Conception to ongoing pregnancy: the 'black box' of early pregnancy loss. Hum. Reprod. Update 8, 333-343 (2002).

5. Greaney, J., Wei, Z. \& Homer, H. Regulation of chromosome segregation in oocytes and the cellular basis for female meiotic errors. Hum. Reprod. Update 24, 135-161 (2017).

6. Fragouli, E. et al. Cytogenetic analysis of human blastocysts with the use of FISH, CGH and aCGH: scientific data and technical evaluation. Hum. Reprod. 26, 480-490 (2011).

7. Vanneste, E. et al. Chromosome instability is common in human cleavage-stage embryos. Nat. Med. 15, 577-583 (2009).

8. van Echten-Arends, J. et al. Chromosomal mosaicism in human preimplantation embryos: a systematic review. Hum. Reprod. Update 17, 620-627 (2011)

9. Verlinsky, Y. et al. Preimplantation diagnosis of common aneuploidies by the first- and second-polar body FISH analysis. J. Assist Reprod. Genet 15, 285-289 (1998)

10. Schattman, G. L. Preimplantation genetic testing for aneuploidy: It's déjà vu all over again! Fertil. Steril. 112, 1046-1047 (2019).

11. Paulson, R. J. Outcome of in vitro fertilization cycles with preimplantation genetic testing for aneuploidies: let's be honest with one another. Fertil. Steril. 112, 1013-1014 (2019).

12. Munné, S. et al. Preimplantation genetic testing for aneuploidy versus morphology as selection criteria for single frozen-thawed embryo transfer in good-prognosis patients: a multicenter randomized clinical trial. Fertil. Steril. 112, 1071-1079.e7 (2019).

13. Gleicher, N. et al. A single trophectoderm biopsy at blastocyst stage is mathematically unable to determine embryo ploidy accurately enough for clinical use. Reprod. Biol. Endocrinol. 15, 33 (2017).

14. Zamani Esteki, M. et al. In vitro fertilization does not increase the incidence of de novo copy number alterations in fetal and placental lineages. Nat. Med. 25, 1699-1705 (2019).

15. Centers for Disease Control and Prevention. 2016 Assisted Reproductive Technology National Summary Report (US Dept of Health and Human Services, 2018).

16. Patrizio, P. et al. Worldwide live births following the transfer of chromosomally 'abnormal' embryos after PGT/A: results of a worldwide web-based survey. J. Assist. Reprod. Genet. 36, 1599-1607 (2019).

17. Greco, E., Minasi, M. G. \& Fiorentino, F. Healthy babies after intrauterine transfer of mosaic aneuploid blastocysts. N. Engl. J. Med. 373, 2089-2090 (2015).

18. Dimitriadou, E. et al. Principles guiding embryo selection following genome-wide haplotyping of preimplantation embryos. Hum. Reprod. 32, 687-697 (2017).

19. Bolton, H. et al. Mouse model of chromosome mosaicism reveals lineage-specific depletion of aneuploid cells and normal developmental potential. Nat. Commun. 7, 1-12 (2016).

20. Eggan, K. et al. Male and female mice derived from the same embryonic stem cell clone by tetraploid embryo complementation. Nat. Biotechnol. 20, 455-459 (2002)

21. Mashiko, D. et al. Chromosome segregation error during early cleavage in mouse pre-implantation embryo does not necessarily cause developmental failure after blastocyst stage. Sci. Rep. 10, 854 (2020).

22. Bond, D. J. \& Chandley, A. C. Aneuploidy (Oxford Univ. Press, 1983).

23. Santaguida, S., Tighe, A., D’Alise, A. M., Taylor, S. S. \& Musacchio, A. Dissecting the role of MPS1 in chromosome biorientation and the spindle checkpoint through the small molecule inhibitor reversine. J. Cell Biol. 190, 73-87 (2010).

24. Santaguida, S. et al. Chromosome mis-segregation generates cell-cycle-arrested cells with complex karyotypes that are eliminated by the immune system. Dev. Cell 41, 638-651.e5 (2017)

25. He, Q. et al. Chromosomal instability-induced senescence potentiates cell non-autonomous tumourigenic effects. Oncogenesis 7, 1-18 (2018).

26. Capo-chichi, C. D., Cai, K. Q., Testa, J. R., Godwin, A. K. \& Xu, X.-X. Loss of GATA6 leads to nuclear deformation and aneuploidy in ovarian cancer. Mol. Cell. Biol. 29, 4766-4777 (2009).

27. Warmflash, A., Sorre, B., Etoc, F., Siggia, E. D. \& Brivanlou, A. H. A method to recapitulate early embryonic spatial patterning in human embryonic stem cells. Nat. Methods 11, 847-854 (2014). 
28. Minn, K. T. et al. High-resolution transcriptional and morphogenetic profiling of cells from micropatterned human embryonic stem cell gastruloid cultures. eLife 9, e59445 (2020)

29. Krendl, C. et al. GATA2/3-TFAP2A/C transcription factor network couples human pluripotent stem cell differentiation to trophectoderm with repression of pluripotency. Proc. Natl Acad. Sci. USA 114, E9579-E9588 (2017).

30. Meinhardt, G. et al. Pivotal role of the transcriptional co-activator YAP in trophoblast stemness of the developing human placenta. Proc. Natl Acad. Sci. USA 117, 13562-13570 (2020).

31. Etoc, F. et al. A balance between secreted inhibitors and edge-sensing controls gastruloid self-organization. Dev. Cell 39, 302-315 (2016).

32. Singla, S., Iwamoto-Stohl, L. K., Zhu, M. \& Zernicka-Goetz, M. Autophagy-mediated apoptosis eliminates aneuploid cells in a mouse model of chromosome mosaicism. Nat. Commun. 11, 2958 (2020).

33. Zaveri, L. \& Dhawan, J. Cycling to meet fate: connecting pluripotency to the cell cycle. Front. Cell Dev. Biol. 6, 57 (2018).

34. Petropoulos, S. et al. Single-cell RNA-seq reveals lineage and X chromosome dynamics in human preimplantation embryos. Cell 165, 1012-1026 (2016).

35. Zhou, F. et al. Reconstituting the transcriptome and DNA methylome landscapes of human implantation. Nature 572, 660-664 (2019).

36. Griffiths, J. A., Scialdone, A. \& Marioni, J. C. Mosaic autosomal aneuploidies are detectable from single-cell RNAseq data. BMC Genomics 18, 904 (2017).

37. Starostik, M. R., Sosina, O. A. \& McCoy, R. C. Single-cell analysis of human embryos reveals diverse patterns of aneuploidy and mosaicism. Genome Res 30, 814-825 (2020).
38. Kalousek, D. K. \& Dill, F. J. Chromosomal mosaicism confined to the placenta in human conceptions. Science 221, 665-667 (1983).

39. Biesecker, L. G. \& Spinner, N. B. A genomic view of mosaicism and human disease. Nat. Rev. Genet. 14, 307-320 (2013).

40. Grati, F. R. et al. Outcomes in pregnancies with a confined placental mosaicism and implications for prenatal screening using cell-free DNA. Genet. Med. 22, 309-316 (2020).

41. Amor, D. J. et al. Health and developmental outcome of children following prenatal diagnosis of confined placental mosaicism. Prenat. Diagn. 26, 443-448 (2006)

42. Kennerknecht, I., Krämer, S., Grab, D., Terinde, R. \& Vogel, W. A prospective cytogenetic study of third-trimester placentae in small-for-date but otherwise normal newborns. Prenat. Diagn. 13, 257-269 (1993).

43. Fryburg, J. S., Dimaio, M. S., Yang-Feng, T. L. \& Mahoney, M. J. Follow-up of pregnancies complicated by placental mosaicism diagnosed by chorionic villus sampling. Prenat. Diagn. 13, 481-494 (1993).

44. Kasak, L., Rull, K., Vaas, P., Teesalu, P. \& Laan, M. Extensive load of somatic CNVs in the human placenta. Sci. Rep. 5, 8342 (2015).

45. Simunovic, M. et al. A 3D model of a human epiblast reveals BMP4-driven symmetry breaking. Nat. Cell Biol. 21, 900-910 (2019).

Publisher's note Springer Nature remains neutral with regard to jurisdictional claims in published maps and institutional affiliations.

(c) The Author(s), under exclusive licence to Springer Nature Limited 2021, corrected publication 2021 


\section{Methods}

Ethics statement. Individuals undergoing embryo transfers at the Center for Human Reproduction, New York (CHR) with PGT-A showing chromosomal-abnormal designated embryos, in addition to routine IVF consent, signed an additional informed consent since 2014, specifically advising them of the hypothetically increased risks that such transfers may lead to chromosomal-abnormal ongoing pregnancies and/or miscarriages and mandating early prenatal diagnosis by CVS or amniocentesis. Anonymized clinical data extraction for this study was approved by the Institutional Review Board of the CHR (ER03302015/01).

Participant selection and transfer of diagnosed aneuploid embryos. Embryo transfers occurred at the CHR. As the CHR does not recommend PGT-A, almost all embryos in this study were produced at other IVF centres, where they were cryopreserved. Embryos with reported trisomies of chromosomes 13, 18 or 21 or sex chromosome abnormalities were not transferred unless patients insisted on such transfers, in which case they were asked to sign an additional 'against medical advice' consent. Other trisomies were transferred after genetic counselling and followed a routine protocol. The morphology of the transfered embryos were graded by the Garden score ${ }^{46}$. Transfer results from 32 individuals with ages ranging from 27 to 47 were analysed. Patient and IVF cycle characteristics including patient number and age are presented in Supplementary Table 2. Their initial chromosomal analyses were performed by national PGT-A laboratories, using next-generation sequencing and, in a few cases, comparative genomic hybridization (Supplementary Table 2), following guidelines of the Preimplantation Genetic Diagnosis International Society ${ }^{47}$. Pregnancy outcomes were determined by routine CVS or amniocentesis in later first or second trimesters of pregnancy.

Cell culture. Human ES cells (registered RUES2 human ES cell line NIHhESC-09-0013) were grown in mouse embryonic fibroblast-conditioned HUESM medium (MEF-CM) supplemented with $20 \mathrm{ng} \mathrm{ml}^{-1}$ basic fibroblast growth factor (bFGF). The culture medium was changed daily. Cells were tested for mycoplasma infection before beginning experiments and again at two-monthly intervals. The tissue culture dishes that cells were grown on were coated with Geltrex (Life Technologies) solution. The dishes were coated overnight at $4{ }^{\circ} \mathrm{C}$ and then incubated at $37^{\circ} \mathrm{C}$ for at least $20 \mathrm{~min}$ before the cells were plated. Cells were passaged using Gentle Cell Dissociation Reagent (Stem Cell Technologies) and replated in MEF-CM with $10 \mu \mathrm{M}$ ROCK inhibitor Y-27632 (Abcam). For reversine treatment, cells that were approximately $75 \%$ confluent were treated with $0.5 \mu \mathrm{M}$ reversine (Cayman Chemicals) in MEF-CM media supplemented with $20 \mathrm{ng} \mathrm{ml}^{-1}$ bFGF with a medium change after $24 \mathrm{~h}$

DAPI staining of chromosome metaphase spreads from RUES2 cells. To obtain chromosomes at the mitotic stage, we treated RUES2 cells with colchicine (Sigma) at a final concentration of $0.3 \mu \mathrm{g} \mathrm{ml}^{-1}$ for $2 \mathrm{~h}$ in the culture medium before collecting them. Cells were dissociated from the culture dish with StemPro Accutase (Life Technologies) and then resuspended in $50 \mu 1$ 1.0\% sodium citrate solution (in distilled water) and incubated on ice for $5 \mathrm{~min}$. To spread the chromosomes, $5 \mu \mathrm{l}$ cell suspension was loaded onto each slide by pulling the pipette tip from one side of the slide to the other while pushing the cell suspension out of the tip. Fixative solution (3:1 methanol:acetic acid, Sigma) was immediately added onto each slide in a drop by drop manner. The slides were air-dried thoroughly and then pre-wetted in PBS supplemented with $0.5 \%(\mathrm{v} / \mathrm{v})$ Photo-Flo (Kodak) and $1 \%(\mathrm{w} / \mathrm{v})$ bovine serum album (Sigma) for $15 \mathrm{~min}$ before staining. The slides were stained with $200 \mu \mathrm{l} 4 \mu \mathrm{M}$ DAPI (Thermo Fisher Scientific) for $5 \mathrm{~min}$ at room temperature and then washed twice with PBS supplemented with $0.5 \%(\mathrm{v} / \mathrm{v})$ Photo-Flo for $5 \mathrm{~min}$ and once with distilled water for $5 \mathrm{~min}$. ProLong Gold antifade mounting medium (Thermo Fisher Scientific) was used to mount coverslips onto slides. G-banding karyotyping was conducted by a commercial service from Cell Line Genetics.

Flow cytometry of aneuploid cells. To analyse the cells obtained after reversine treatment $(0.5 \mathrm{mM}$ for $24 \mathrm{~h})$, we used the two-camera ImageStream $(\mathrm{X})$ imaging flow cytometer. The control and reversine-treated RUES2 samples, 263,000 and 67,500 cells, respectively, were stained with DAPI and collected on ImageStream $\mathrm{X}$ (Amnis). The instrument combines the high capacity and immunofluorescent sensitivity and capacity of a flow cytometer with high-resolution microscopy and high-content image analysis pipelines. Images were captured using a $\times 40$ objective and with INSPIRE acquisition software (Amnis v.6.2). Post-acquisition data analysis was performed using IDEAS software (Amnis v.6.2). Single cells in focus were gated using Gradient RMS (focus), then plots of area versus aspect ratio of the bright-field channel were used to select the main cluster of cells to be further analysed. Next, the intensity and area of the DAPI channel were used to characterize the two populations. The intensity feature was used as input for the cell cycle module of IDEAS.

Generation of cell-tracking RUES2 lines. Two cell-tracking human ES cell lines (VENUS-H2B-RUES2 and RFP-H2B-RUES2) were generated from the parental RUES2 line using the ePiggyBac system. Accordingly, ePiggyBac plasmids were designed. To generate RFP-H2B-RUES2 line, we nucleofected the cells with an
ePiggyBac plasmid containing RFP-H2B driven by the CAG promoter and also containing a blasticidin resistance (BSD) gene (ePB-BSD-CAG-RFP-H2B). Cells were then selected with $5 \mu \mathrm{g} \mathrm{ml}^{-1}$ BSD. To generate the VENUS-H2B-RUES2 line, we nucleofected the cells with an ePiggyBac plasmid containing H2B-VENUS driven by the CAG promoter and also containing a puromycin resistance (Puro) gene (ePB-Puro-CAG-H2B-VENUS). Cells were selected with $1 \mu \mathrm{g} \mathrm{ml}^{-1}$ of puromycin. Cells that survived selection were passaged as single cells using StemPro Accutase (Life Technologies), plated in MEF-CM supplemented with $10 \mathrm{mM}$ ROCK inhibitor and allowed to grow into colonies. Individual colonies were picked and plated into separate wells with growth medium and ROCK inhibitor. Each clone was isolated and controlled for normal karyotype (G-banding) and pluripotency maintenance.

Micropattern culture and gastruloid differentiation. The culture chips for RUES2 to form gastruloids were made using micropatterned glass coverslips (Arena A and Arena EMB A) from Cytoo (Cambridge). The micropatterns were coated with $800 \mathrm{ml}$ of $10 \mu \mathrm{g} \mathrm{ml}^{-1}$ recombinant laminin 521 (BioLamina) in PBS with calcium and magnesium (PBS++) for $3 \mathrm{~h}$ at $37^{\circ} \mathrm{C}$ before cell plating. The micropatterned coverslips were stored in $35 \mathrm{~mm}$ plastic tissue culture dished and then serially washed with PBS++ to remove laminin. After two washes with PBS, RUES2 cells were dissociated from the culture dish with StemPro Accutase (Life Technologies) for $5 \mathrm{~min}$ and resuspended in MEF-CM with $10 \mu \mathrm{M}$ ROCK inhibitor. Eight-hundred-thousand cells in $2 \mathrm{ml}$ of MEF-CM with $20 \mathrm{ng} \mathrm{ml}^{-1} \mathrm{bFGF}$, $100 \mu \mathrm{g} \mathrm{ml}^{-1}$ Normocin (Invivogen) and ROCK inhibitor were seeded in each cultured dish containing one micropatterned coverslip. The ROCK inhibitor was removed from the medium $3 \mathrm{~h}$ after seeding and cells were incubated overnight $(12-18 \mathrm{~h})$ before BMP4 induction. To induce gastruloid differentiation, the cells grown on the micropattern in the culture dish were cultured with $2 \mathrm{ml}$ induction medium (MEF-CM supplemented with $20 \mathrm{ng} \mathrm{ml}^{-1} \mathrm{bFGF}, 100 \mu \mathrm{g} \mathrm{ml}^{-1}$ normocin and $50 \mathrm{ng} \mathrm{ml}^{-1} \mathrm{BMP} 4$ (R\&D systems)) for $48 \mathrm{~h}$.

Immunofluorescence. Cells were washed once with PBS and then fixed with $4 \%$ paraformaldehyde (Electron Microscopy Sciences) for $30 \mathrm{~min}$. After three washes for $5 \mathrm{~min}$ with PBS, samples were blocked and permeabilized with blocking buffer (PBS supplemented with 3\% normal donkey serum (Jackson Immunoresearch) with $0.3 \%$ Triton X-100 (Sigma)) for $30 \mathrm{~min}$. Cells were then incubated with primary antibodies diluted in blocking buffer at room temperature for $2 \mathrm{~h}$ and followed by three washes with PBS. Secondary antibodies conjugated to Alexa 488, Alexa 555, Alexa 594 or Alexa 647 (1:1,000 dilution, Molecular Probes for mouse, rabbit and goat; Thermo Fisher Scientific) and $10 \mathrm{ng} \mathrm{ml}^{-1}$ DAPI were added for $30 \mathrm{~min}$ before three washes with PBS. The coverslips were then mounted on to glass slides using ProLong Gold antifade mounting medium (Invitrogen) for imaging. Primary antibodies used are listed in Supplementary Table 3.

Imaging and image analysis. Chromosome spreads were imaged under a microscope (Olympus BX51) with a $\times 100$ oil-immersion objective. Image process and chromosome counts were performed with ImageJ (FIJI). All micropattern confocal images were acquired on a Zeiss Inverted LSM 780 laser scanning confocal microscope with a $\times 10, \times 20$ or $\times 40$ water-immersion objective. Quantification of fluorescent intensity and area fraction was performed by ImageJ (FIJI) using the Analyse/Measure command. Quantification for cell numbers on the micropattern colonies was performed with Ilastik and ImageJ. In brief, for segmentation of individual cells, we first used Ilastik classification to separate foreground from background. The classifier was trained for each experiment on the DAPI images of four randomly chosen colonies from that experiment. The cell counting was then conducted using the Analyze/Analyze Particles command in ImageJ. Quantification of the cell-tracking line for different cell fates was also performed in ImageJ using the colocalization plugin for ComDet version 0.4.1. The quantification of the radial area of ectoderm (SOX2+) of each colony was quantified using custom software written in Matlab. Live-cell-tracking imaging for the cell-tracking RUES2 lines that generated Supplementary Video 1 was performed with a spinning-disc microscope (CellVoyager CV1000, Yokogawa), acquired using the CellVoyager CV1000 spinning disk confocal system (Yokagawa/Olympus) with a $\times 20$ dry objective, deconvolved using Autoquant software, and analysed in Imaris.

Aneuploidy detection from scRNA-seq. To detect the aneuploidy rate in human embryos, we analysed two published scRNA-seq datasets, which contain 1,529 cells from 88 E3-E7 embryos ${ }^{34}$ and 5,911 cells from 21 E6-E14 embryos ${ }^{35}$. These two sets of data were analysed separately to avoid the bias induced by the technical variance. The method applied uses chromosome-wide expression imbalances to identify aneuploidies from scRNA-seq data as previously described ${ }^{36}$, which provides quantitative aneuploidy calls. In brief, expression level (expressed as counts per million) was normalized the by the median of the gene across cells, and highly expressed genes (median > 50 counts) were selected. For each chromosome and cell, expression levels across genes were summed. The sum was normalized by the number of considered genes on each chromosome to make it comparable across chromosomes. Within each cell, we convert this normalized sum into a score $(S$-score) centred at 1 across chromosomes. If no chromosome within a cell has gain or loss of copy number, this score will deviate randomly around 1. Otherwise, 
their scores will be increased or decreased accordingly. To demonstrate whether a chromosome displays aberrant copy number, we then converted this score into a $Z$-score where the variance was estimated separately for each chromosome across cells using the median absolute deviation. We defined cells with any chromosome expression profile above $|3|$ to be putatively aneuploid. Aneuploid chromosomes were identified using a false discovery rate-corrected $P<0.1$, where the $P$-value was obtained using Student's $t$-distribution. To call an aneuploidy, we not only required that the corrected $P$-value was less than 0.1 but also imposed an effect-size threshold such that cell-chromosomes where $0.8<S<1.2$ were not considered significant.

Statistical analysis and reproducibility. No statistical methods were used to predetermine sample size. The gastruloid experiments were randomized as each cell colony analysed was randomly selected from both control and experimental groups. Each experiment was repeated independently at least three times with similar results, unless otherwise stated. The investigators were not blinded to allocation during experiments and outcome assessment. Unbiased analysis of data was carried out wherever possible. All statistical procedures performed are indicated in the figure captions. $P$-values are calculated using Student's $t$-test.

Reporting Summary. Further information on research design is available in the Nature Research Reporting Summary linked to this article.

\section{Data availability}

Publicly available expression matrices of human embryo single-cell RNA-seq data were obtained from ArrayExpress E-MTAB-3929 and the Gene Expression Omnibus (accession number GSE109555). All other data supporting the findings of this study are available from the corresponding author upon reasonable request. Source data are provided with this paper.

\section{Code availability}

For the chromosome-wide analysis of aneuploidy in human embryos using scRNA-seq data, a strategy similar the one described in ref. ${ }^{36}$ was used. The specific code used for these data is available upon request. Source data are provided with this paper.

\section{References}

46. Gardner, D. K., Lane, M., Stevens, J., Schlenker, T. \& Schoolcraft, W. B. Blastocyst score affects implantation and pregnancy outcome: towards a single blastocyst transfer. Fertil. Steril. 73, 1155-1158 (2000).
47. Preimplantation Genetic Diagnosis Society. Position Statement on Chromosome Mosaicism and Preimplantation Aneuploidy Testing at the Blastocyst Stage (PGDIS, 2016); http://pgdis.org/docs/newsletter_071816.html

\section{Acknowledgements}

This work was supported by private funds provided by the Foundation for Reproductive Medicine to the Center for Human Reproduction, and private funds from The Rockefeller University. M.Y. was supported by a fellowship from the Lalor Foundation. We are particularly grateful to the patients who moved their embryos for transfer to $\mathrm{CHR}$. We thank our colleagues at CHR and members of the Brivanlou Laboratory for their advice and constructive criticism; A. North for access and advice to the Rockefeller Core Imaging Facility; S. Mazel and S. Semova of the Flow Cytometry Research Center; and J.-M. Santel and A. Souza for their assistance in preparing this manuscript under the challenging COVID-19 situation in New York City.

\section{Author contributions}

M.Y., T.R., N.G. and A.H.B. conceived the project. M.Y., T.R. and J.N performed all gastruloid experiments and M.Y. conducted quantification analysis. T.R. performed the flow cytometry experiment. J.M. and T.R. conducted the aneuploidy analysis of the scRNA-seq data. M.Y. and J.H. performed the karyotyping chromosome-spreading experiment. D.H.B. and N.G. retrieved and analysed the patient data. M.Y. organized the patient data. R.S. and D.F.A. contributed to the result interpretation and manuscript writing. M.Y., T.R. and A.H.B. wrote the manuscript. All authors discussed the results and reviewed the manuscript.

\section{Competing interests}

N.G. and A.H.B. are co-founders of OvaNova Inc. A.H.B. is a co-founder of Rumi Scientific Inc. The other authors declare no competing interests.

\section{Additional information}

Extended data is available for this paper at https://doi.org/10.1038/s41556-021-00660-7. Supplementary information The online version contains supplementary material available at https://doi.org/10.1038/s41556-021-00660-7.

Correspondence and requests for materials should be addressed to A.H.B. or N.G. Peer review information Nature Cell Biology thanks Joris Vermeesch and the other, anonymous, reviewers for their contribution to the peer review of this work.

Reprints and permissions information is available at www.nature.com/reprints. 

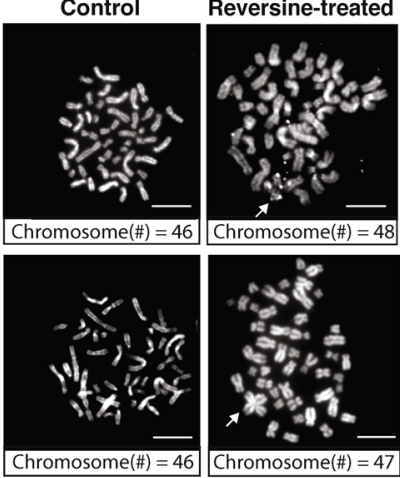

b

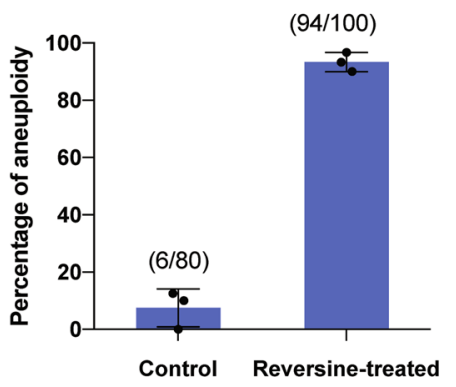

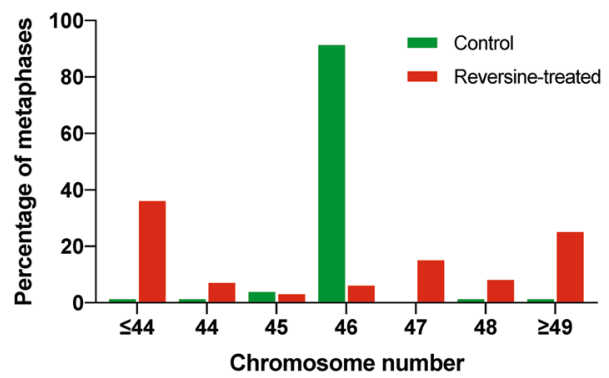

d
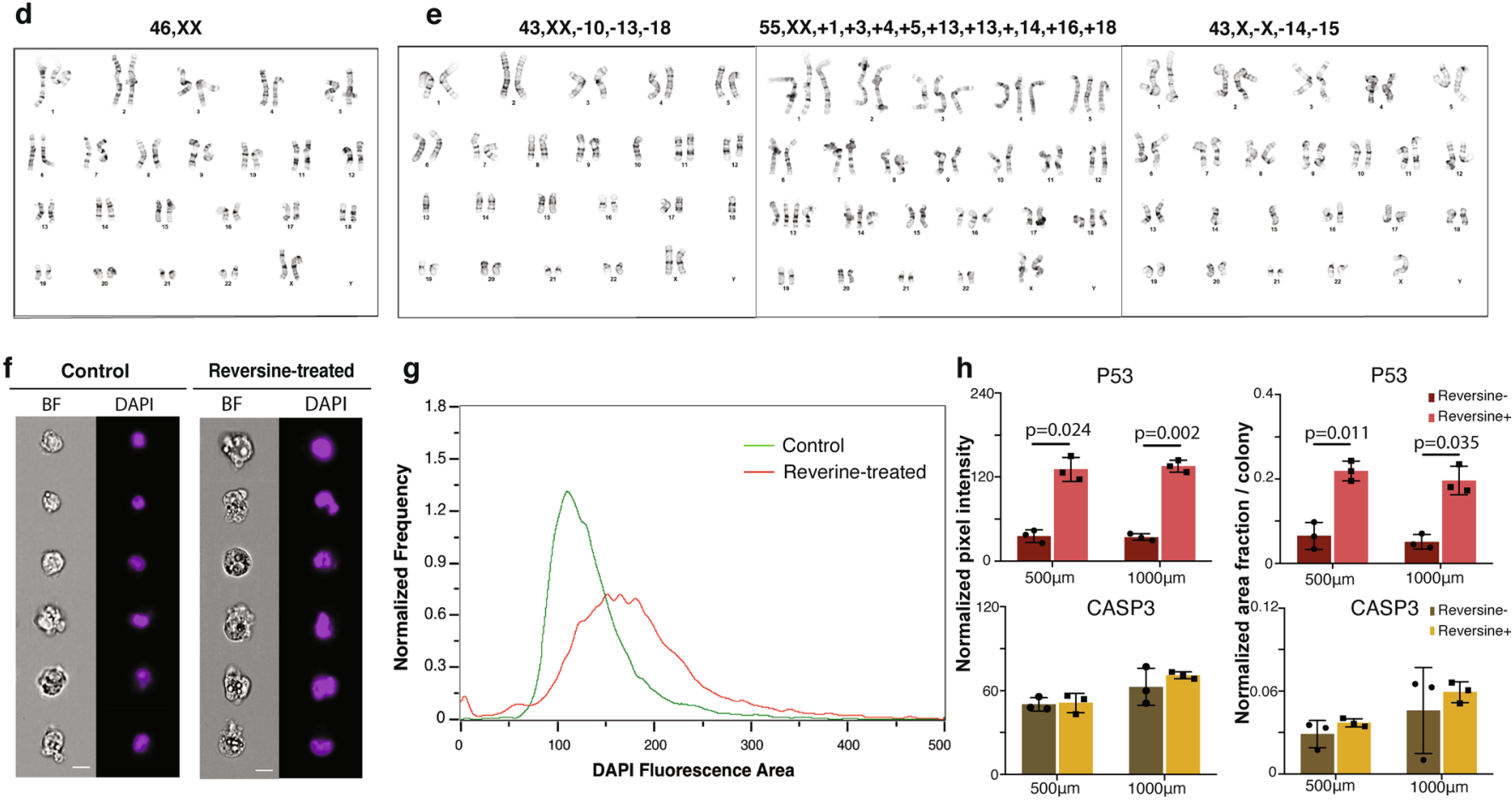

Extended Data Fig. 1 | Reversine treatment induces aneuploidy in RUES2. a, Representative images of metaphase spreads stained with DAPI of reversine-treated and untreated control RUES2. RUES2 treated with reversine ( $24 \mathrm{~h}$ ) have abnormal chromosome numbers. Arrows indicate chromosome fragmentation or abnormal crossover. b. Percentage of aneuploid cells detected in reversine-treated ( 80 cells) and control RUES2 (100 cells). $n=3$ independent experiment replicates. c, Chromosome counts from metaphase spreads of reversine-treated ( 80 cells) and control cells ( 100 cells). d, G-banding karyotype of untreated RUES2 cell line. $\mathbf{e}$, G-banding karyotypes of reversine-treated RUES2 cells. $\mathbf{f}$, Representative cell images of reversine-treated and untreated control RUES2 taken by imaging flow cytometry. Reversine-treated RUES2 exhibit different nuclear (DAPI) morphology than control RUES2. BF, bright field. $\mathbf{g}$, Plot of DNA content (mean nuclear area; DAPI) obtained from flow cytometry of reversine-treated and untreated RUES2. Compared to control, reversine-treated RUES2 displays larger nuclei. $\mathbf{h}$, Normalized fluorescent pixel intensity and area fraction were quantified for P53 and CASP3 on micropatterned colonies after $48 \mathrm{hr}$ culture in pluripotency medium. Reversine-treated cell colonies have a significant higher level of P53 in both fluorescence intensity and area fraction compared with untreated colonies. CASP3 level has no significant difference between treated and control groups. $\mathrm{N}=3$ biologically independent cell colonies Results are shown as the mean $\pm \mathrm{s}$.d. and statistical significance was calculated using a two-tailed Student's t-test. For $\mathbf{a}$ and $\mathbf{f}$, scale bar $=10 \mu \mathrm{M}$. 
a
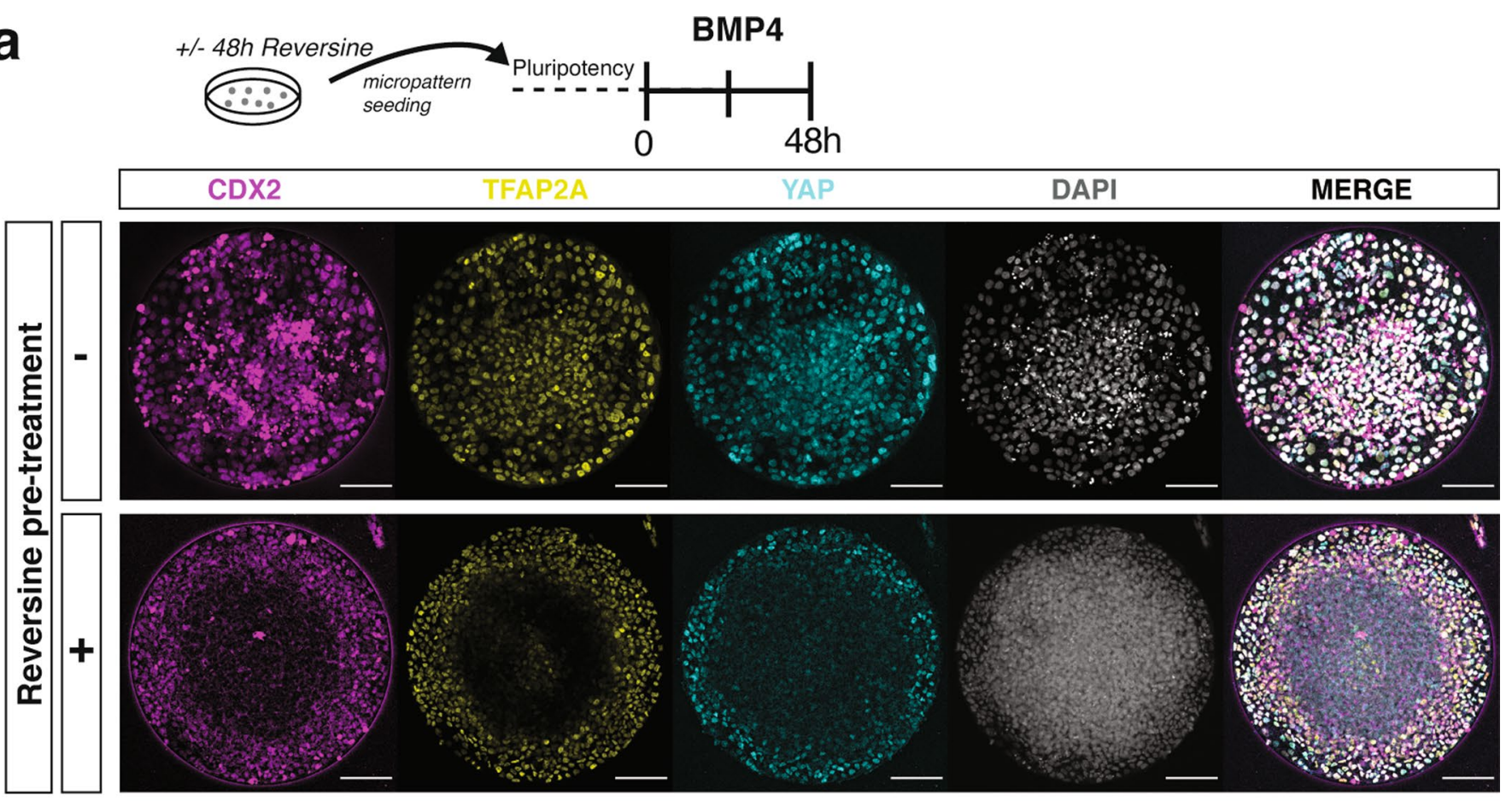

b
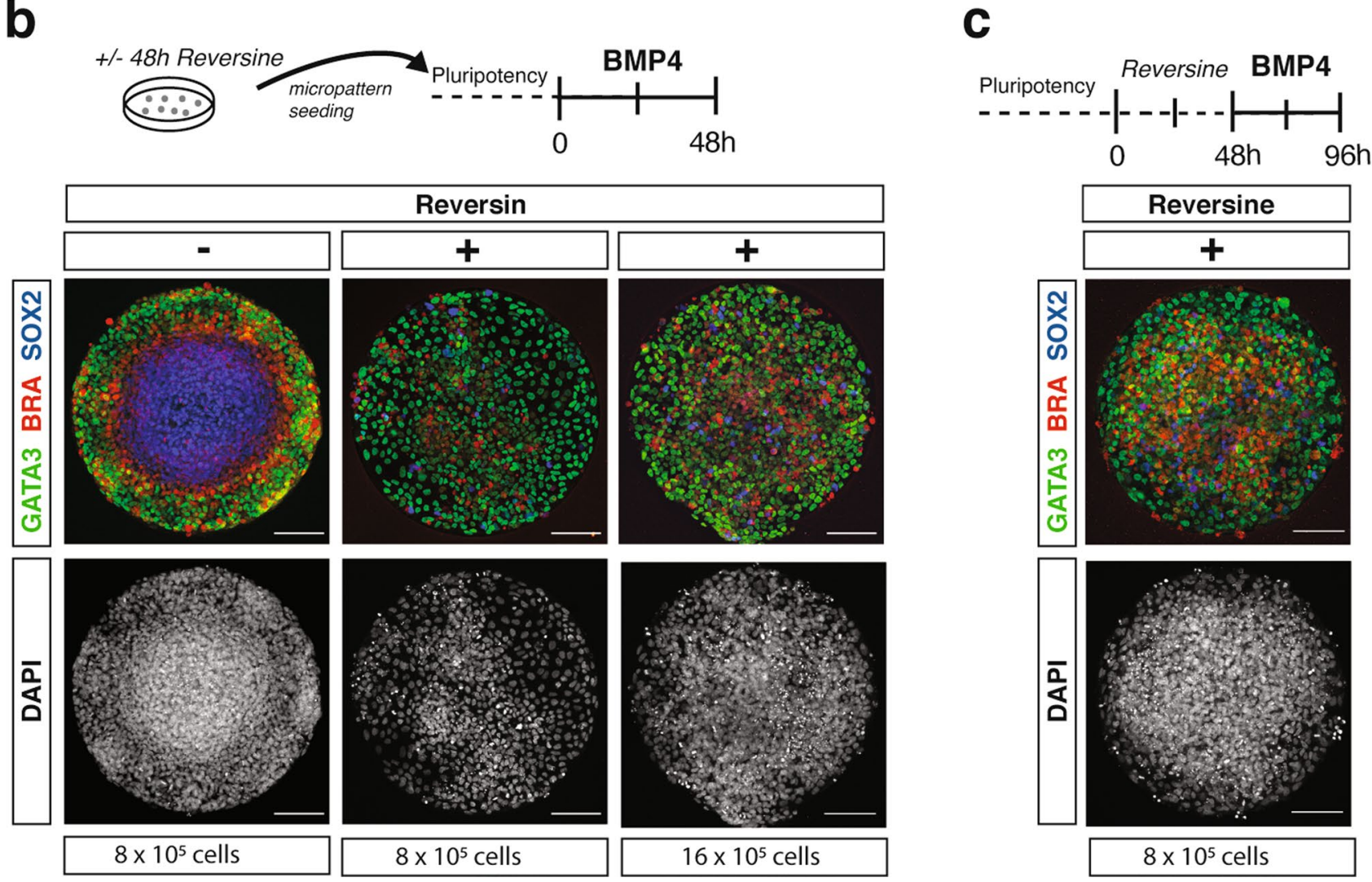

Extended Data Fig. 2 | Depletion of embryonic cell fate domain in aneuploid gastruloids is density-independent. a, RUES2 (standard amount; $8 \times 10^{5}$ ) were seeded on laminin-coated micropatterns and cultured with $50 \mathrm{ng} / \mathrm{ml} \mathrm{BMP4}$ for 48 hr to generate gastruloids. The cells in gastruloids derived from reversine-treated RUES2 express CDX2, TFAP2A at the protein-level and show nuclear YAP staining consistent with it acquiring a trophectoderm/ amnion fate. $\mathbf{b}$, Standard $\left(8 \times 10^{5}\right)$ or twice the standard amount of reversine-treated aneuploid RUES2 was seeded on laminin-coated micropatterns and cultured with $50 \mathrm{ng} / \mathrm{ml} \mathrm{BMP4}$ to form gastruloids. The gastruloids derived from aneuploid RUES2 with both cell concentrations are depleted of the ectoderm center (SOX2+) and mesoderm ring (BRA+). c, RUES2 were seeded and treated with $0.5 \mu \mathrm{M}$ reversine for $48 \mathrm{hr}$ on the micropatterns before BMP4 induction. Direct reversine treatment on the micropatterns allows the aneuploid cells to proliferate to the ideal density before BMP4 induction. The gastruloids derived are still depleted of SOX $2+$ and BRA+ domains. For a-c, scale bar $=100 \mu \mathrm{m}$. Only $500 \mu \mathrm{m}$ micropattern substrates are shown. 


\section{Reporting Summary}

Nature Research wishes to improve the reproducibility of the work that we publish. This form provides structure for consistency and transparency in reporting. For further information on Nature Research policies, see our Editorial Policies and the Editorial Policy Checklist.

\section{Statistics}

For all statistical analyses, confirm that the following items are present in the figure legend, table legend, main text, or Methods section.

n/a Confirmed

$\square$ The exact sample size $(n)$ for each experimental group/condition, given as a discrete number and unit of measurement

$\square$ \ A statement on whether measurements were taken from distinct samples or whether the same sample was measured repeatedly

$\square$ The statistical test(s) used AND whether they are one- or two-sided

$\square$ Only common tests should be described solely by name; describe more complex techniques in the Methods section.

\ $\square$ A description of all covariates tested

Х $\square$ A description of any assumptions or corrections, such as tests of normality and adjustment for multiple comparisons

$\triangle$ A full description of the statistical parameters including central tendency (e.g. means) or other basic estimates (e.g. regression coefficient)

AND variation (e.g. standard deviation) or associated estimates of uncertainty (e.g. confidence intervals)

For null hypothesis testing, the test statistic (e.g. $F, t, r$ ) with confidence intervals, effect sizes, degrees of freedom and $P$ value noted Give $P$ values as exact values whenever suitable.

Х $\square$ For Bayesian analysis, information on the choice of priors and Markov chain Monte Carlo settings

Х $\square$ For hierarchical and complex designs, identification of the appropriate level for tests and full reporting of outcomes

Х $\square$ Estimates of effect sizes (e.g. Cohen's $d$, Pearson's $r$ ), indicating how they were calculated

Our web collection on statistics for biologists contains articles on many of the points above.

\section{Software and code}

Policy information about availability of computer code

Data collection Chromosome metaphase spread imaging: Olympus BX51 with a 100x oil-immersion objective.

Micropattern Immunofluorescent confocal imaging: Zeiss Inverted LSM 780 laser scanning confocal microscope with a $10 x, \times 20$ or $\times 40$ waterimmersion objective.

Time lapse imaging: CellVoyager CV1000 spinning disk confocal system (Yokagawa/Olympus) with a 20x dry objective, deconvolved using Autoquant software.

Flow cytometry: two-camera ImageStream (X) imaging flow cytometer (40X) with INSPIRE acquisition software (Amnis v.6.2).

Data analysis

Quantification of fluorescent intensity and area fraction was performed by ImageJ (FIJI, v 2.0.0) using Analyse/Measure command. Quantification for cell numbers on the micropattern colonies was performed with llastik (1.3.3rc2) and ImageJ.

The cell counting was then conducted using the Analyze/Analyze Particles command in ImageJ. Quantification of the cell tracking line for different cell fates was also performed in ImageJ using the colocalization plugin for ComDet version 0.4.1.

The quantification of the radial area of ectoderm (SOX2+) of each colony was quantified using custom software written in Matlab ( $r 2016 a)$.

Time lapse imaging was deconvolved using Autoquant software (X 2.2.0), and analysed in Imaris (9.1.0).

Cytometry images were analysed with INSPIRE acquisition software (Amnis v.6.2). Post-acquisition data analysis was performed using IDEAS software (Amnis v.6.2). The intensity feature was used as input for the cell cycle module of IDEAS.

Statistical analysis was carried out and using GraphPad Prism (v 8.0).

Figures were assembled in Adobe Illustrator (CC 2020).

ScRNA Data was analysed in R (v4.0.2) and ggplot2 (v3); code will be available upon request. 
Policy information about availability of data

All manuscripts must include a data availability statement. This statement should provide the following information, where applicable:

- Accession codes, unique identifiers, or web links for publicly available datasets

- A list of figures that have associated raw data

- A description of any restrictions on data availability

Publicly available expression matrices of single-cell RNA-seq were obtained from: ArrayExpress E-MTAB-3929 and GEO (accession number GSE109555).

\section{Field-specific reporting}

Please select the one below that is the best fit for your research. If you are not sure, read the appropriate sections before making your selection. Х Life sciences Behavioural \& social sciences Ecological, evolutionary \& environmental sciences

For a reference copy of the document with all sections, see nature.com/documents/nr-reporting-summary-flat.pdf

\section{Life sciences study design}

All studies must disclose on these points even when the disclosure is negative.

Sample size Statistical or power analysis is not appropriate for estimating sample numbers used to demonstrate cellular differentiation potential because pilot data on which to base these analyses is absent and, even small numbers of appropriately integrated cells over several (3) repeated experiments would be a significant and high impact finding.

Data exclusions No data was excluded from the analysis.

Replication All replicates were successful. Analysis of a minimum of 3 individual micro-patterned colonies from at least three independent experiments (different seeding and cell batch/passage) were performed.

Randomization The gastruloid experiments were randomized as each cell colony analysed was randomly selected from both control and experimental groups.

Blinding The same investigator set up and analysed the experiments, no blinding was performed. Unbiased analysis of data was carried out whereever possible

\section{Reporting for specific materials, systems and methods}

We require information from authors about some types of materials, experimental systems and methods used in many studies. Here, indicate whether each material, system or method listed is relevant to your study. If you are not sure if a list item applies to your research, read the appropriate section before selecting a response.

Materials \& experimental systems

\begin{tabular}{l|l}
\hline$n / a$ & Involved in the study \\
\hline & $\bigotimes$ Antibodies \\
\hline & $\bigotimes$ Eukaryotic cell lines \\
$\square$ & $\square$ Palaeontology and archaeology \\
$\square$ & $\square$ Animals and other organisms \\
$\square$ & $\square$ Human research participants \\
$\triangle$ & $\square$ Clinical data
\end{tabular}

\begin{tabular}{l|l}
\multicolumn{2}{l}{ Methods } \\
\hline n/a & Involved in the study \\
$\square$ & $\square$ ChIP-seq \\
$\square$ & $\bigotimes$ Flow cytometry \\
$\square$ & $\square$ MRI-based neuroimaging
\end{tabular}

\section{Antibodies}

Antibodies used

SOX2 (Cell Signaling \#3579S rabbit mAb), 1:200

BRACHYURY (R\&D Systems \#AF2085 goat polyAb), 1:300

GATA3 (Thermo Fisher Scientific \#MA1-028 mouse mAb), 1:2000CT4 (R\&D Systems \#MAB1759 mouse mAb), 1:400

NANOG (R\&D Systems \#AF1997 PolyAB), 1:200

Active CASPASE 3 (R\&D Systems \#AF835 Rabbit polyAB), 1:500

P53 (Thermo Fisher Scientific \#MA5-12557 mouse mAb), 1:300

CDX2 (R\&D Systems \#AF3665 goat polyAB), 1:200

TFAP2A (DSHB 3B5 mouse mAb), 1:200

YAP (Cell Signaling \#4912S Rabbit mAB), 1:200

Donkey anti-Rabbit lgG (H+L) Highly Cross-Adsorbed Secondary Antibody, Alexa Fluor 488 (Thermo Fisher Scientific, \#A21206), 1:1000 
Donkey anti-Goat IgG $(\mathrm{H}+\mathrm{L})$ Cross-Adsorbed Secondary Antibody, Alexa Fluor 555 (Thermo Fisher Scientific, \#A21432), 1:1000

Donkey anti-Rabbit IgG (H+L) Highly Cross-Adsorbed Secondary Antibody, Alexa Fluor 594 (Thermo Fisher Scientific, \#A21207), 1:1000

Donkey anti-Mouse IgG (H+L) Highly Cross-Adsorbed Secondary Antibody, Alexa Fluor 647 (Thermo Fisher Scientific, \#A31571),1:1000

Validation

All antibodies used were validated by the manufactures from which they were purchased from. Based on the validation statement on the maufacturer's websites, the antibodies were titrated, optimized and assessed for expected cellular localization.

\section{Eukaryotic cell lines}

Policy information about cell lines

Cell line source(s)

RUES2 (NIH\#0013) background cell lines were procured from the RU Stem Cell Core facility.

This line was created in our lab and are listed in the NIH Human Embryonic Stem Cell Registry. They were derived under approval from the Tri-Institutional Stem Cell Initiative Embryonic Stem Cell Research Oversight (Tri-SCI ESCRO) Committee, an independent committee charged with oversight of research with human pluripotent stem cells and embryos to ensure conformance with University policies, and guidelines from the U.S. National Academy of Sciences (NAS)

Authentication

All cell lines used in this study were authenticated by karyotype. Fluorescent lines were validated by imaging and sequencing. Pluripotency of all hES lines was confirmed by expression of OCT4, SOX2, and NANOG, as well as by successful generation of gastruloids in BMP4 culture.

Mycoplasma contamination

All cell lines were tested and confirmed to be mycoplasma free before the onset of experiments. Cell lines were tested at bimonthly intervals through the course of the experiments.

Commonly misidentified lines

(See ICLAC register)

\section{None}

\section{Human research participants}

Policy information about studies involving human research participants

Population characteristics

In this study reported clinical care was provided at The Center for Human Reproduction (CHR) n New York City as part of routine clinical care. Here presented data were extracted from the center's anonymized electronic research data bank. Patients included in this study were suffering from infertility and had been treated with in vitro fertilization, which included embryonic testing for aneuploidy (preimplantation genetic testing for aneuploidy, PGTA) at a variety of commercial nationa PGT-A laboratories that revealed no euploid embryos. They, therefore, after genetic counseling, elected to have selected embryos with alleged aneuploid embryo transferred. Embryo transfer results of 32 patients were analyzed. The maternal age of the patients range from 27 to 47 .

Recruitment

There was no active recruitment for this study. Presented data are based on a retroactive review of clinical care at The CHR

Ethics oversight

Anonymized data extraction for this study was approved by the IRB of the Center for Human Reproduction (ER03302015/01).

Note that full information on the approval of the study protocol must also be provided in the manuscript.

\section{Flow Cytometry}

Plots

Confirm that:

\The axis labels state the marker and fluorochrome used (e.g. CD4-FITC).

\The axis scales are clearly visible. Include numbers along axes only for bottom left plot of group (a 'group' is an analysis of identical markers).

$\bigotimes$ All plots are contour plots with outliers or pseudocolor plots.

$\bigotimes$ A numerical value for number of cells or percentage (with statistics) is provided.

Methodology

Sample preparation

Instrument

Software

Cell population abundance

Gating strategy
Cells were fixed in 4\% PFA and stained with DAPI.

ImageStream X (Amnis).

INSPIRE acquisition and IDEAS softwares (Amnis v.6.2)

RUES2 hESCs control sample: 263,000 cells, and Reversine-treated sample: 67,500 cells.

Only DAPI (nucleus staining) was used in the analyzed samples for visualizing the nuclei. Therefore, there is no positive and negative population. Cells in focus were chosen with Gradient RMS and single cells were selected from scatter plots of the bright-field Area versus Aspect Ratio as shown in supplementary. Fig. 1.

$\bigotimes$ Tick this box to confirm that a figure exemplifying the gating strategy is provided in the Supplementary Information. 